\title{
Observation of Enhanced Double Parton Scattering in Proton-Lead Collisions at $\sqrt{s_{N N}}=8.16 \mathrm{TeV}$
}

\author{
R. Aaij et al. \\ (LHCb Collaboration)
}

(Received 13 July 2020; revised 4 September 2020; accepted 14 October 2020; published 20 November 2020)

\begin{abstract}
A study of prompt charm-hadron pair production in proton-lead collisions at $\sqrt{s_{N N}}=8.16 \mathrm{TeV}$ is performed using data corresponding to an integrated luminosity of about $30 \mathrm{nb}^{-1}$, collected with the $\mathrm{LHCb}$ experiment. Production cross sections for different pairs of charm hadrons are measured and kinematic correlations between the two charm hadrons are investigated. This is the first measurement of associated production of two charm hadrons in proton-lead collisions. The results confirm the predicted enhancement of double parton scattering production in proton-lead collisions compared to the single parton scattering production.
\end{abstract}

DOI: 10.1103/PhysRevLett.125.212001

At high-energy hadron colliders, particles are produced in fundamental collisions of internal partons in the beam projectiles. The underlying parton densities are described by parton distribution functions (PDFs). A collision event can produce multiple heavy-flavor hadrons via a single parton scattering (SPS) or multiple parton scatterings. The latter, generating on average a larger number of charged tracks, could explain the heavy-flavor production rate in high-multiplicity events [1-4]. In a simple model, assuming that the PDFs of two partons in the same projectile are independent, the associated production cross section of final-state particles $A$ and $B$ from two separate partonic interactions, i.e., a double parton scattering (DPS) process, is related to the inclusive production cross section of $A$ and $B, \sigma^{A}$ and $\sigma^{B}$, as [5-14],

$$
\sigma_{\mathrm{DPS}}^{A B}=\frac{1}{1+\delta_{A B}} \frac{\sigma^{A} \sigma^{B}}{\sigma_{\mathrm{eff}}}
$$

Here, $\delta_{A B}=1$ if $A$ and $B$ are identical and is zero otherwise, and $\sigma_{\text {eff }}$ is the so-called effective cross section. The parameter $\sigma_{\text {eff }}$ is related to the collision geometry and is expected to be independent of the final state [15-17]. In proton-ion collisions, following the Glauber model [18], SPS production cross section is expected to scale with the ion mass number in the absence of nuclear matter effects. However, DPS production is enhanced compared to a mass number scaling due to collisions of partons from two

\footnotetext{
*Full author list given at the end of the article.

Published by the American Physical Society under the terms of the Creative Commons Attribution 4.0 International license. Further distribution of this work must maintain attribution to the author(s) and the published article's title, journal citation, and DOI. Funded by SCOAP ${ }^{3}$.
}

different nucleons in the ion, and the enhancement factor is about three in proton-lead $(p-\mathrm{Pb})$ collisions $[10,19-25]$.

The production of two open charm hadrons, $D_{1} D_{2}$, and $J / \psi D$ meson pairs is of particular interest in the study of SPS and DPS processes, as the cross section is relatively large and the high charm-quark mass permits perturbative calculations even at low transverse momentum $\left(p_{T}\right)$. In this Letter, $D$ and $D_{1,2}$ refer to either a $D^{0}, D^{+}$, or $D_{s}^{+}$meson and the inclusion of charge conjugate states is implied. Both like-sign (LS) and opposite-sign (OS) open charm hadron pairs are considered. In an LS pair the two hadrons have the same charm-quark flavor, while in an OS pair they have opposite charm flavors. Pairs of OS charm hadrons can be produced from a $c \bar{c}$ pair via SPS, thus the kinematics of the two hadrons are correlated, while DPS produces correlated and uncorrelated OS pairs. The correlation in SPS production may be modified in heavy-ion data compared to proton-proton $(p p)$ collisions, due to nuclear matter effects [26-33]. The OS correlation is predicted to be sensitive to the properties of the hot medium formed in ultrarelativistic heavy nucleus-nucleus collisions [34-44].

The two hadrons in an LS pair produced in a DPS process are expected to be uncorrelated. Studies of LS pair production and correlation in different environments help to test the universality of the parameter $\sigma_{\text {eff }}$ and gain insight into the underlying parton correlations [45]. Since DPS production involves two parton pairs, it is very sensitive to the nuclear PDF (NPDF) in proton-ion collisions, including its possible dependence on the position inside the nucleus [46].

Production of OS charm and beauty pairs has been studied in fully reconstructed decays [47-51] and using partially reconstructed decays [52-59], and the hadron and antihadron are found to be correlated; in particular, the azimuthal angle $\Delta \phi$ between the two hadron directions projected to the plane transverse to the beam line favors 
values close to $\Delta \phi=0$ or $\pi$. Production of LS charm pairs, double quarkonium and multiple jets at the Tevatron and the LHC revealed evidence of DPS signals [51,60-68]. The effective cross section is measured to be in the range of 10 to $20 \mathrm{mb}$ for most final states, however, a value as low as $5 \mathrm{mb}$ is extracted using double quarkonium production [69-71]. More measurements are required to resolve this puzzle.

This Letter presents the first measurement of charm pair production in proton-lead collisions at a nucleon-nucleon center-of-mass energy of $\sqrt{s_{N N}}=8.16 \mathrm{TeV}$. The data were collected with the LHCb experiment at a low interaction rate in two distinct beam configurations. In the $p \mathrm{~Pb}$ configuration, particles produced in the direction of the proton beam are analyzed, while in the $\mathrm{Pb} p$ configuration particles are analyzed in the $\mathrm{Pb}$ beam direction. The $p \mathrm{~Pb}(\mathrm{~Pb} p)$ data correspond to an integrated luminosity of $12.2 \pm 0.3 \mathrm{nb}^{-1} \quad\left(18.6 \pm 0.5 \mathrm{nb}^{-1}\right)$. The detector coordinate system is defined to have the $z$ axis aligned with the proton beam direction. In the following, particle rapidities $(y)$ are defined in the nucleon-nucleon rest frame.

The $\mathrm{LHCb}$ detector is a single-arm forward spectrometer described in detail in Refs. [72,73]. The online event selection is performed by a trigger, which consists of a hardware stage, based on information from the calorimeter and muon systems, followed by a software stage, which applies a full event reconstruction. Charm hadrons $\left(H_{c} \equiv D^{0}, D^{+}, D_{s}^{+}, J / \psi\right)$ are reconstructed online via the decays $D^{0} \rightarrow K^{-} \pi^{+}, D^{+} \rightarrow K^{-} \pi^{+} \pi^{+}, D_{s}^{+} \rightarrow K^{-} K^{+} \pi^{+}$, and $J / \psi \rightarrow \mu^{+} \mu^{-}$. The data samples are selected by the hardware trigger based on the calorimeter activity for $D$ candidates and based on the muon system for $J / \psi$ candidates. Candidate pairs are formed by $D^{0} D^{0}, D^{0} \bar{D}^{0}$, and $D^{+} D^{ \pm}$combinations (same species), and $D^{0} D^{ \pm}$, $D^{0} D_{s}^{ \pm}, D^{+} D_{s}^{ \pm}$, and $J / \psi D^{0,+}$ combinations (different species). Other charm pairs are not considered due to their limited yield in the data. The tracks used to reconstruct the $D$ mesons are required to be positively identified as kaons or pions and must be separated from every primary $p-\mathrm{Pb}$ collision vertex (PV). These tracks are also required to have $p_{T}>250 \mathrm{MeV} / c$ and at least one track must have $p_{T}>$ $500 \mathrm{MeV} / c\left(p_{T}>1000 \mathrm{MeV} / c\right)$ for $D^{0}\left(D^{+}, D_{s}^{+}\right)$final states. The tracks are required to form a vertex of good quality that is separated from every PV. The reconstructed $D$ mesons are required to be consistent with originating from a PV, which favors prompt production over mesons from beauty-hadron decays (denoted as charm-from- $b$ ). The two muons used to reconstruct $J / \psi$ candidates are required to have $p_{T}>500 \mathrm{MeV} / c$ and form a goodquality vertex.

In the off-line selection, kaons and pions are required to have momentum $p>3 \mathrm{GeV} / c$, and muons to have $p>6 \mathrm{GeV} / c, p_{T}>750 \mathrm{MeV} / c$ and be positively identified by using information from all subdetectors [74,75].
The $K^{-} K^{+}$invariant mass from the $D_{s}^{+} \rightarrow K^{-} K^{+} \pi^{+}$decay is required to be within $\pm 20 \mathrm{MeV} / c^{2}$ of the known $\phi(1020)$ mass [76]. A kinematic fit is performed on each charm hadron and on the pair, constraining them to originate from a PV. Requirements on the fit qualities strongly reduce charm-from- $b$ contributions but retain more than $99 \%$ of prompt pairs.

Results are obtained in a charm-hadron kinematic region $p_{T}\left(H_{c}\right)<12 \mathrm{GeV} / c$ and $1.7<y\left(H_{c}\right)<3.7$ $\left(-4.7<y\left(H_{c}\right)<-2.7\right)$ for $p \mathrm{~Pb}(\mathrm{~Pb} p)$ data. For $D^{+}$and $D_{s}^{+}$mesons the requirement $p_{T}\left(H_{c}\right)>2 \mathrm{GeV} / c$ is applied due to extremely small yields at lower $p_{T}$. Total cross sections of $D^{0} D^{0}, D^{0} \bar{D}^{0}$, and $J / \psi D^{0}$ pair production are also evaluated in the full $\mathrm{LHCb}$ rapidity acceptance, $1.5<$ $y\left(H_{c}\right)<4\left(-5<y\left(H_{c}\right)<-2.5\right)$ for $p \mathrm{~Pb}(\mathrm{~Pb} p)$ data, in order to compare with single charm production [77,78].

The cross section for a charm pair is calculated as $\sigma=N^{\text {corr }} /\left(\mathcal{L} \times \mathcal{B}_{1} \times \mathcal{B}_{2}\right)$, where $\mathcal{L}$ is the integrated luminosity, and $N^{\text {corr }}$ is the signal yield after efficiency correction and the subtraction of charm-from- $b$ background. The branching fractions of the two charm-hadron decays $\mathcal{B}_{1,2}$ are taken from Ref. [76] for the $D^{0}, D^{+}, J / \psi$ decays, and $\mathcal{B}\left[D_{s}^{+} \rightarrow\left(K^{+} K^{-}\right)_{\phi} \pi^{+}\right]=(2.24 \pm 0.13) \%$ from Refs. [79,80]. The raw signal yield is determined from an unbinned maximum likelihood fit to the distribution of the invariant masses $m_{1}$ and $m_{2}$ of the two charm hadrons. The two-dimensional probability densities comprise four components: signal-signal, background-background, signal-background, and background-signal for the first-second charm hadron in a pair. The background is mainly from random combinations of tracks. The signal component for each charm hadron is described by the sum of a Gaussian and a Crystal Ball function [81] and the background component by an exponential function. The distribution for pairs of same-species hadrons is constructed to be independent of the ordering of $m_{1}$ and $m_{2}$. As an example, the $\left(m_{1}, m_{2}\right)$ distribution for $D^{0} D^{0}$ candidates and its projection on $m_{1}$ and $m_{2}$ are shown in Fig. 1 for $p \mathrm{~Pb}$ data, with the fit projections overlaid. More distributions are shown in the Supplemental Material [82]. The raw signal yield is between 100 and 4000 for all hadron pairs considered.

The total detection efficiency for each individual charm hadron is evaluated from simulated signal decays, properly corrected using control samples of $p-\mathrm{Pb}$ collisions. These control samples are used to calibrate track finding and particle identification (PID) efficiencies [83]. In the simulation, minimum-bias $p$ - $\mathrm{Pb}$ collisions are produced using the EPOS generator [84] according to beam configurations of the data. Charm hadrons are generated in $p p$ collisions at $\sqrt{s}=8.16 \mathrm{TeV}$ using PyTHIA8 $[85,86]$ and are embedded into EPOS minimum-bias events. Particle decays are described by EvTGEN [87], while the particle interaction with the detector, and its response, are implemented using the GEANT4 toolkit [88] as described in Ref. [89]. The track 

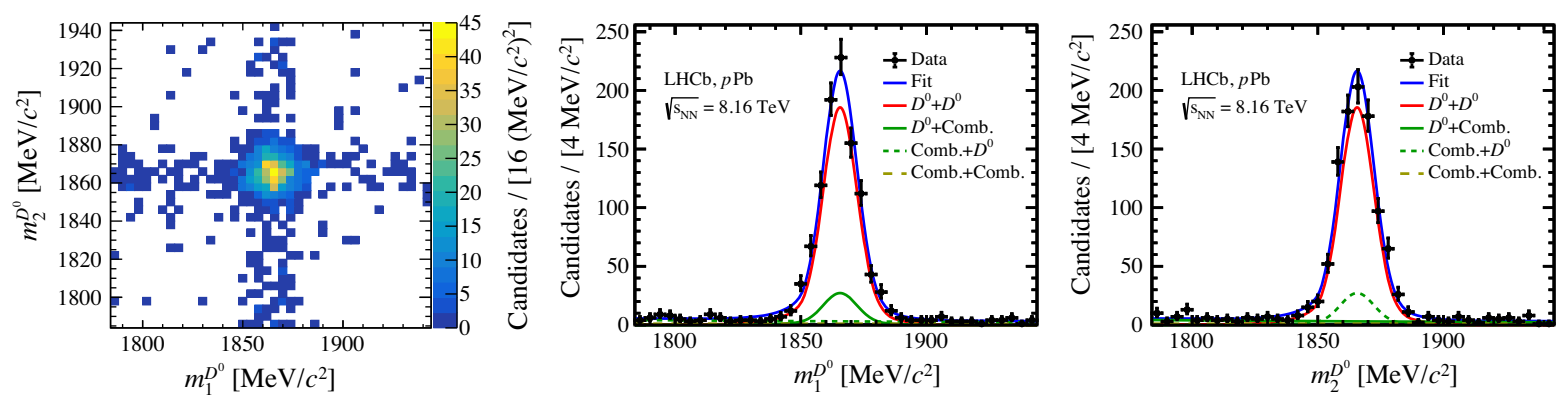

FIG. 1. Two-dimensional invariant-mass distributions of $\left(m_{1}, m_{2}\right)$ for $D^{0} D^{0}$ pairs and the projections on $m_{1}$ and $m_{2}$ are shown on the left, in the middle and on the right respectively, with the fit results superimposed. Shown in the projection plots are (points with bars) $p \mathrm{~Pb}$ data, (solid blue) the total fit and its four components.

finding efficiency in data and simulation is studied with a tag-and-probe method using $J / \psi \rightarrow \mu^{+} \mu^{-}$decays [90]. Similarly, the PID efficiency is measured using large control samples of $D^{0} \rightarrow K^{-} \pi^{+}$and $J / \psi \rightarrow \mu^{+} \mu^{-}$decays for $K^{-}, \pi^{+}$, and $\mu^{-}$tracks, in bins of track momentum and pseudorapidity $(p, \eta)$. The average charged-track multiplicity in OS data is similar to the one in the control samples, while for LS data it is about 13\% higher, which is consistent with a larger contribution of multiple parton scattering in LS data [1-4]. The corresponding difference in detector occupancy results in different detection efficiencies in LS and OS data, which is evaluated in control samples. Efficiencies from control samples are combined with simulation to obtain the efficiency for each charm hadron as a function of $p_{T}$ and $y, \epsilon\left(p_{T}, y\right)$, which is used to determine the efficiency corrected signal yield $\sum_{i}\left[w^{i} / \epsilon_{1}\left(p_{T}^{i}, y^{i}\right) \epsilon_{2}\left(p_{T}^{i}, y^{i}\right)\right]$. Here, $w^{i}$ is the signal sPlot weight [91] used to remove the contribution of background and is obtained from the fit to the invariant-mass distribution, and $\epsilon_{1,2}\left(p_{T}{ }^{i}, y^{i}\right)$ is the efficiency for the first and second hadron in the $i$ th candidate pair in data. The signal yield is then corrected for the charm-from- $b$ contamination, which is estimated to be less than $1 \%$ for open charm pairs and $(4 \pm 2) \%((3.0 \pm 1.5) \%)$ for $J / \psi D$ pairs in $p \mathrm{~Pb}$ $(\mathrm{Pb} p)$ data.

Several sources of systematic uncertainties are investigated. The variation of the signal yield is studied with fits to the invariant-mass distribution using a different signal or background model. A maximum relative variation of $2 \%$ is obtained on the signal yield. The dominant systematic uncertainty arises from the limited control sample size to determine the track finding efficiency, which is on average about 5\% (10\%) per track in $p \mathrm{~Pb}(\mathrm{~Pb} p)$ data. An uncertainty of $2 \%$ per hadron track is introduced to account for the loss of particles due to interactions with the detector material. Because of the small sample size and the choice of $(p, \eta)$ binning for each track, the PID efficiencies obtained from control samples introduce an uncertainty of less than $1 \%$ on the total efficiency of each charm hadron. Other contributions include the uncertainty on the total efficiency due to the size of the simulation sample, the uncertainty on the charm decay branching fractions, the uncertainty on the luminosity measurements and on the charm-from- $b$ fraction. These uncertainties are propagated to the cross-section measurements.

Total cross sections are determined for all charm pairs. Results are detailed in the Supplemental Material [82]. For LS open charm pairs, the measurements are in good agreement with theoretical calculations including both SPS and DPS production [24]. The $J / \psi D^{0}$ cross section is found to be generally higher than SPS production, calculated using the weighted EPPS16 NPDF [92-95].

Prompt single charm cross sections in $p \mathrm{~Pb}$ data were measured to be smaller than those of $\mathrm{Pb} p$ data $[78,96]$, which is explained by modifications of the NPDF. The same effect would result in even stronger suppression of DPS production in $p \mathrm{~Pb}$ compared to $\mathrm{Pb} p$ data due to the participation of two pairs of partons. For charm pairs, the cross-section ratio between $p \mathrm{~Pb}$ and $\mathrm{Pb} p$ data, the forward-backward ratio $\left(R_{\mathrm{FB}}\right)$, is determined for $2.7<$ $\left|y\left(H_{c}\right)\right|<3.7, p_{T}\left(H_{c}\right)>2 \mathrm{GeV} / c$, to be $0.40 \pm 0.05 \pm$ $0.10(0.61 \pm 0.04 \pm 0.12)$ averaged over LS (OS) open charm pairs, and is $0.26 \pm 0.06 \pm 0.04$ for $J / \psi D$ pairs. Here and in the following, the first uncertainty is statistical and the second is systematic. The results indicate reduced production in $p \mathrm{~Pb}$ compared to $\mathrm{Pb} p$ data for both $\mathrm{LS}$ and OS pairs. The $R_{\mathrm{FB}}$ of OS production is compatible with that of prompt $D^{0}$ mesons $[78,96]$, while that of LS production is smaller. The ratio between the $R_{\mathrm{FB}}$ of $\mathrm{LS}$ and $\mathrm{OS}$ production, $0.66 \pm 0.09 \pm 0.03$, is in good agreement with the $R_{\mathrm{FB}}$ of OS data and the $R_{\mathrm{FB}}$ of prompt $D^{0}$ production. The measurements favor the interpretation of LS production via DPS.

The LS over OS cross-section ratio, $R^{D_{1} D_{2}} \equiv$ $\sigma^{D_{1} D_{2}} / \sigma^{D_{1} \bar{D}_{2}}$, is determined for all studied $D_{1} D_{2}$ pairs under the $p_{T}(D)>2 \mathrm{GeV} / c$ requirement, giving an average value of $0.308 \pm 0.015 \pm 0.010$ and $0.391 \pm 0.019 \pm$ 0.025 for $p \mathrm{~Pb}$ and $\mathrm{Pb} p$ data, respectively. The measurements agree with the calculations in Ref. [24] of $0.57_{-0.41}^{+0.16}$ $(p \mathrm{~Pb})$ and $0.52_{-0.38}^{+0.17}(\mathrm{~Pb} p)$, and are significantly larger than that in $p p$ collisions where $R^{D^{0} D^{0}}=0.109 \pm 0.008$ [51], indicating an enhancement of LS pair production over OS 

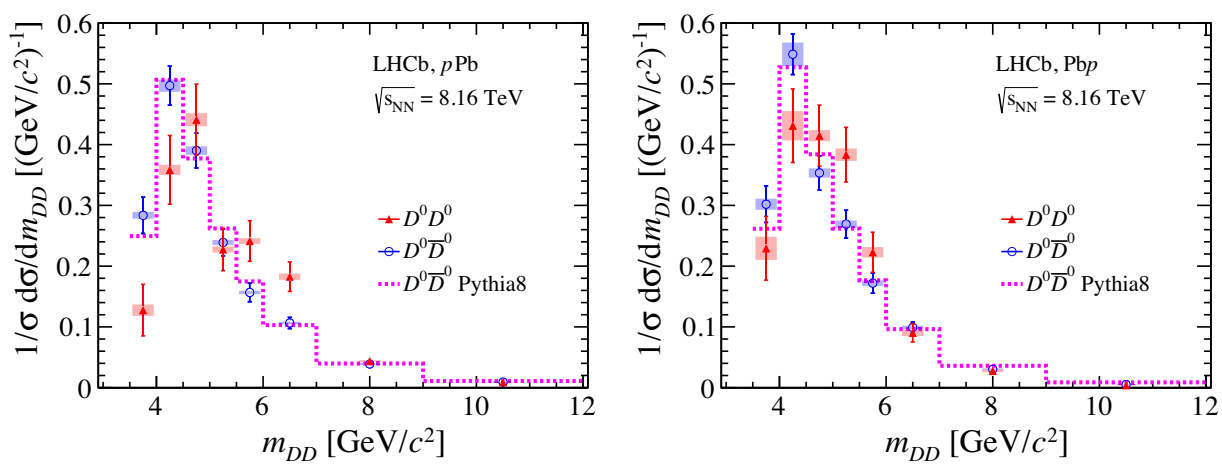

FIG. 2. Two-charm hadron invariant-mass distribution of (red) $D^{0} D^{0}$ and (blue) $D^{0} \bar{D}^{0}$ pairs in (left) $p \mathrm{~Pb}$, (right) $\mathrm{Pb} p$ data and (magenta dashed line) PYTHIA8 simulation. Vertical bars (filled box) are statistical (systematic) uncertainties.

pairs in $p-\mathrm{Pb}$ collisions. The differential results as a function of $y\left(H_{c}\right)$ is shown in the Supplemental Material [82].

The correlations of kinematics between the two charm hadrons in a pair are investigated from the distributions of the two-charm invariant mass $\left(m_{D D}\right)$ and their relative azimuthal angle $\Delta \phi$. The differential cross section for each variable is normalized by the total cross section, such that the largest systematic uncertainty, the one from the track finding efficiency, almost completely cancels. As examples, in Fig. 2, the $m_{D D}$ distribution is shown for $D^{0} D^{0}$ and $D^{0} \bar{D}^{0}$ pairs without any requirement on $p_{T}(D)$. The difference between $D^{0} D^{0}$ and $D^{0} \bar{D}^{0}$ pairs is determined to be more than three (two) standard deviations in $p \mathrm{~Pb}$ $(\mathrm{Pb} p)$ data, studied using a $\chi^{2}$ test. For both $D^{0} D^{0}$ and $D^{0} \bar{D}^{0}$ pairs, the $m_{D D}$ distribution is compatible between $p \mathrm{~Pb}$ and $\mathrm{Pb} p$ data. The $D^{0} \bar{D}^{0}$ pair shows a similar $m_{D D}$ distribution to that of the PYTHIA8 simulation, in which the fraction of inclusive charm production that contains more than one charm pair within the $\mathrm{LHCb}$ acceptance is about $7 \%$.

The $\Delta \phi$ distribution is shown in Fig. 3 for $D^{0} D^{0}$ and $D^{0} \bar{D}^{0}$ pairs with and without the requirement $p_{T}\left(D^{0}\right)>2 \mathrm{GeV} / c$. Without this condition, the $\Delta \phi$ distribution is almost uniform for both LS and OS pairs, similar to that in PythiA8 simulation. However, with the
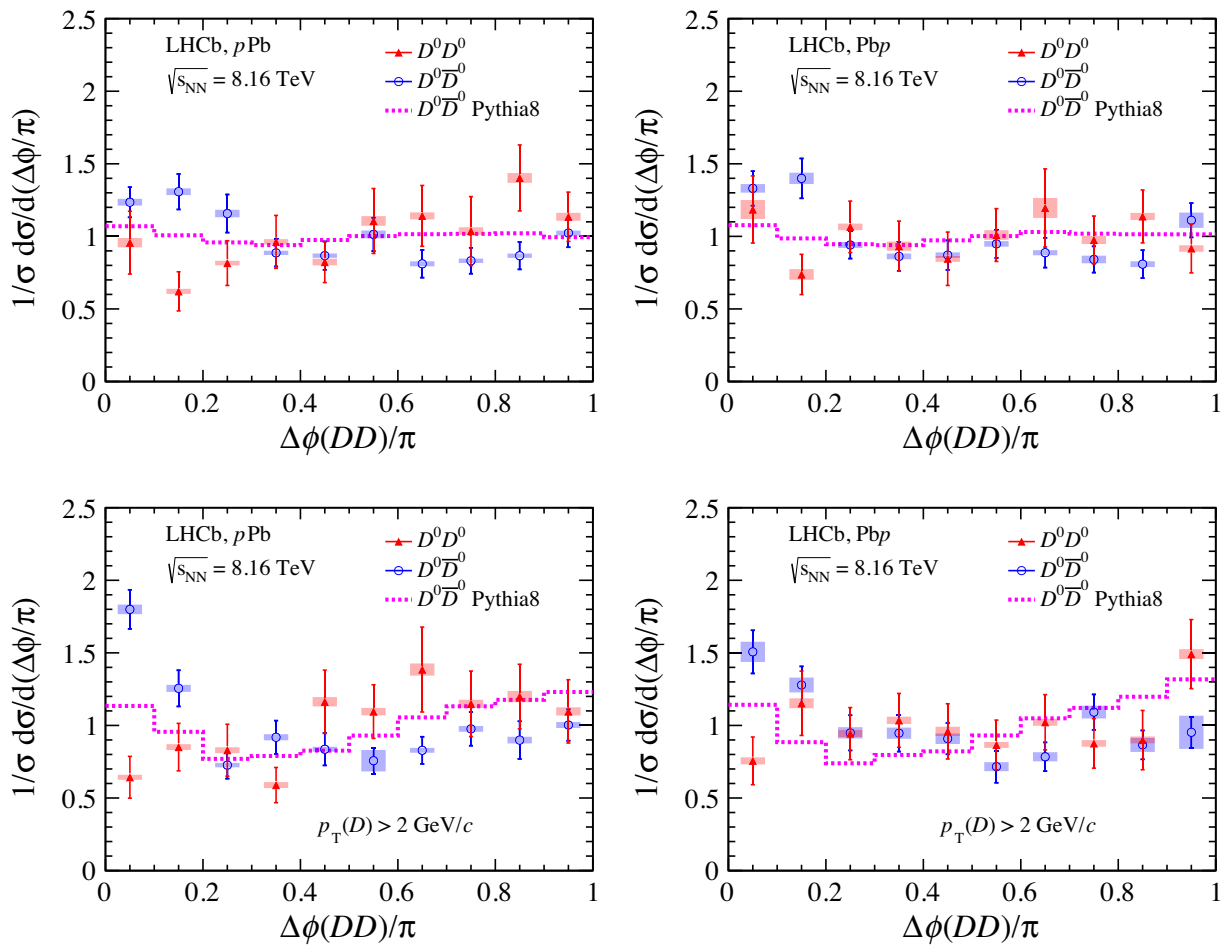

FIG. 3. The $\Delta \phi$ distribution for (red) $D^{0} D^{0}$ and (blue) $D^{0} \bar{D}^{0}$ pairs in (left) $p \mathrm{~Pb}$, (right) $\mathrm{Pb} p$ data and the (magenta dashed line) PYTHIA8 simulation, (bottom) with and (top) without the $p_{T}\left(D^{0}\right)>2 \mathrm{GeV} / c$ requirement. Vertical bars (filled box) are statistical (systematic) uncertainties. 
TABLE I. The effective cross section $\sigma_{\text {eff, } p \mathrm{~Pb}}$ (in $b$ ) measured using $J / \psi D^{0}$ and $D^{0} D^{0}$ pair production in $p$ - $\mathrm{Pb}$ data and the extrapolated values from $p p$ data [80].

\begin{tabular}{lccc}
\hline \hline Pairs & $-5<y\left(H_{c}\right)<-2.5$ & $1.5<y\left(H_{c}\right)<4$ & $p p$ extrapolation \\
\hline$D^{0} D^{0}$ & $0.99 \pm 0.09 \pm 0.09$ & $1.41 \pm 0.11 \pm 0.10$ & $4.3 \pm 0.5$ \\
$J / \psi D^{0}$ & $0.64 \pm 0.10 \pm 0.06$ & $0.92 \pm 0.22 \pm 0.06$ & $3.1 \pm 0.3$ \\
\hline \hline
\end{tabular}

$p_{T}\left(D^{0}\right)>2 \mathrm{GeV} / c$ requirement, the $D^{0} \bar{D}^{0}$ pair favors values $\Delta \phi \sim 0$, while that of $D^{0} D^{0}$ pairs is still compatible with being flat, and both show inconsistency with the PYTHIA8 simulation. In general, the behavior that $m_{D D}$ distribution in $D^{0} D^{0}$ pairs peaks at higher values compared to that of $D^{0} \bar{D}^{0}$ pairs and the flat $D^{0} D^{0} \Delta \phi$ distribution are qualitatively consistent with a large DPS contribution in LS pair production. Distributions of the pair transverse momentum and the two-charm relative rapidity are found to be compatible in OS data, LS data, and the PYTHIA8 simulation.

The effective cross section $\sigma_{\mathrm{eff}, p \mathrm{~Pb}}$ is calculated according to Eq. (1) using the $D^{0} D^{0}$ and $J / \psi D^{0}$ cross sections [6], assuming solely DPS production, where the prompt $J / \psi$ and $D^{0}$ production are evaluated from $\mathrm{LHCb}$ measurements $[77,78]$. The results are displayed in Table I with a typical value of order $1 b$. Table I (" $p p$ extrapolation") also provides the $\sigma_{\text {eff, } p p}$ result [80] scaled by the Pb nucleus mass number 208, which is valid under the assumption of SPS production and absence of nuclear modification. The result confirms the expectation that DPS production in $p-\mathrm{Pb}$ collisions is enhanced by a factor of 3 compared to SPS production, consistent with the expectation from the Glauber model. The $\sigma_{\text {eff }, p \text { Pb }}$ value measured using $J / \psi D^{0}$ production is smaller than that observed in $D^{0} D^{0}$ production, as measured in $p p$ data [80], which may be due to SPS contamination [97] or more than expected $J / \psi D^{0}$ DPS production. The $p \mathrm{~Pb}$ data show a higher $\sigma_{\text {eff, } p \mathrm{~Pb}}$ value compared to $\mathrm{Pb} p$ data, which may suggest a complicated structure of the NPDF, as studied in Ref. [46].

The nuclear modification factor, $R \equiv \sigma_{p \mathrm{~Pb}} / 208 \sigma_{p p^{\prime}}$, is measured for $J / \psi D^{0}$ and $D^{0} D^{0}$ pairs with $R^{H_{c} H_{c}^{\prime}}=$ $R^{H_{c}} \times R^{H_{c}^{\prime}} \times 208 \sigma_{\mathrm{eff}, p p} / \sigma_{\mathrm{eff}, p \mathrm{~Pb}}$, where $\sigma_{p \mathrm{~Pb}}$ and $\sigma_{p p}$ are the cross sections of charm pairs in $p-\mathrm{Pb}$ and $p p$ collisions, respectively. Assuming variations of $R$ and $\sigma_{\text {eff, } p p}$ as a function of collision energy are small for $p_{T}$-integrated production, using measurements of $\sigma_{\text {eff, } p p}$ [51], $R^{J / \psi}$ [77], and $R^{D^{0}}[96], R^{D^{0} D^{0}}=1.3 \pm 0.2(4.2 \pm 0.8)$ and $R^{J / \psi D^{0}}=$ $1.5 \pm 0.5(4.6 \pm 1.3)$ for $p \mathrm{~Pb}(\mathrm{~Pb} p)$ data are obtained, where the uncertainties are the total. The results are about a factor of 3 larger compared to that of single $J / \psi$ or $D^{0}$ hadron production $[77,96]$.

To summarize, the production of LS and OS open charm hadron pairs as well as $J / \psi D$ pairs are studied in $p-\mathrm{Pb}$ collisions at $\sqrt{s_{N N}}=8.16 \mathrm{TeV}$ using fully reconstructed decays. The cross-section ratio between LS and OS pairs is found to be a factor of 3 higher than that in $p p$ data.
The forward-backward ratio of OS pairs is compatible with single charm production, while a smaller value is found for LS pairs. Distributions of the two-charm invariant mass and relative azimuthal angle show a difference between LS and OS pairs, and the LS pairs exhibit a flat relative azimuthal angle distribution independent of charm hadron $p_{T}$. The effective cross-section and nuclear modification factor for $J / \psi D^{0}$ and $D^{0} D^{0}$ are in general compatible with the expected enhancement factor of 3 for DPS over SPS production ratio from $p p$ to $p$ - $\mathrm{Pb}$ collisions. This is the first direct observation of such an enhancement using LS charm production in $p$ - $\mathrm{Pb}$ data. The $\sigma_{\mathrm{eff}, p \mathrm{~Pb}}$ result is different between $p \mathrm{~Pb}$ and $\mathrm{Pb} p$ data and between $J / \psi D^{0}$ and $D^{0} D^{0}$ pairs may suggest additional effects not considered yet, which deserve further investigation using future $\mathrm{LHCb}$ data samples.

We would like to thank Hannu Paukkunen and Huasheng Shao for providing theoretical predictions and helpful discussions. We express our gratitude to our colleagues in the CERN accelerator departments for the excellent performance of the LHC. We thank the technical and administrative staff at the LHCb institutes. We acknowledge support from CERN and from the national agencies: CAPES, CNPq, FAPERJ, and FINEP (Brazil); MOST and NSFC (China); CNRS/IN2P3 (France); BMBF, DFG, and MPG (Germany); INFN (Italy); NWO (Netherlands); MNiSW and NCN (Poland); MEN/IFA (Romania); MSHE (Russia); MinECo (Spain); SNSF and SER (Switzerland); NASU (Ukraine); STFC (United Kingdom); DOE NP and NSF (USA). We acknowledge the computing resources that are provided by CERN, IN2P3 (France), KIT and DESY (Germany), INFN (Italy), SURF (Netherlands), PIC (Spain), GridPP (United Kingdom), RRCKI and Yandex LLC (Russia), CSCS (Switzerland), IFIN-HH (Romania), CBPF (Brazil), PL-GRID (Poland) and OSC (USA). We are indebted to the communities behind the multiple open-source software packages on which we depend. Individual groups or members have received support from AvH Foundation (Germany); EPLANET, Marie Skłodowska-Curie Actions and ERC (European Union); A*MIDEX, ANR, Labex P2IO, and OCEVU, and Région Auvergne-Rhóne-Alpes (France); Key Research Program of Frontier Sciences of CAS, CAS PIFI, and the Thousand Talents Program (China); RFBR, RSF, and Yandex LLC (Russia); GVA, XuntaGal, and GENCAT (Spain); the Royal Society and the Leverhulme Trust (United Kingdom). 
[1] H. Abramowicz et al., Summary of the workshop on multiparton interactions (MPI@LHC 2012), arXiv:1306.5413.

[2] B. Abelev et al. (ALICE Collaboration), $J / \psi$ production as a function of charged particle multiplicity in $p p$ collisions at $\sqrt{s}=7 \mathrm{TeV}$, Phys. Lett. B 712, 165 (2012).

[3] S. Acharya et al. (ALICE Collaboration), Measurement of electrons from heavy-flavour hadron decays as a function of multiplicity in $p-\mathrm{Pb}$ collisions at $\sqrt{s_{N N}}=5.02 \mathrm{TeV}$, J. High Energy Phys. 02 (2020) 077.

[4] P. Bartalini and J. R. Gaunt, Multiple parton interactions at the LHC, Adv. Ser. Dir. High Energy Phys. 29, 1 (2018).

[5] F. Abe et al. (CDF Collaboration), Double parton scattering in $\bar{p} p$ collisions at $\sqrt{s}=1.8 \mathrm{TeV}$, Phys. Rev. D 56, 3811 (1997).

[6] M. H. Seymour and A. Siodmok, Extracting $\sigma_{\text {effective }}$ from the LHCb double-charm measurement, arXiv:1308.6749.

[7] J. R. Gaunt and W. J. Stirling, Double parton distributions incorporating perturbative QCD evolution and momentum and quark number sum rules, J. High Energy Phys. 03 (2010) 005.

[8] J. R. Gaunt, C.-H. Kom, A. Kulesza, and W. J. Stirling, Same-sign $W$ pair production as a probe of double parton scattering at the LHC, Eur. Phys. J. C 69, 53 (2010).

[9] C. H. Kom, A. Kulesza, and W. J. Stirling, Pair Production of $J / \psi$ as a Probe of Double Parton Scattering at LHCb, Phys. Rev. Lett. 107, 082002 (2011).

[10] M. Łuszczak, R. Maciuła, and A. Szczurek, Production of two $c \bar{c}$ pairs in double-parton scattering, Phys. Rev. D 85, 094034 (2012).

[11] S. P. Baranov, A. M. Snigirev, and N. P. Zotov, Double heavy meson production through double parton scattering in hadronic collisions, Phys. Lett. B 705, 116 (2011).

[12] P. Bartalini et al., Multi-parton interactions at the LHC, arXiv:1111.0469.

[13] J.-P. Lansberg, H.-S. Shao, N. Yamanaka, and Y.-J. Zhang, Prompt $J / \psi$-pair production at the LHC: Impact of loopinduced contributions and of the colour-octet mechanism, Eur. Phys. J. C 79, 1006 (2019).

[14] M. Alvioli, M. Azarkin, B. Blok, and M. Strikman, Revealing minijet dynamics via centrality dependence of double parton interactions in proton-nucleus collisions, Eur. Phys. J. C 79, 482 (2019).

[15] M. G. Ryskin and A. M. Snigirev, A fresh look at double parton scattering, Phys. Rev. D 83, 114047 (2011).

[16] D. Treleani, Double parton scattering, diffraction and effective cross section, Phys. Rev. D 76, 076006 (2007).

[17] G. Calucci and D. Treleani, Proton structure in transverse space and the effective cross-section, Phys. Rev. D 60, 054023 (1999).

[18] M. L. Miller, K. Reygers, S. J. Sanders, and P. Steinberg, Glauber modeling in high energy nuclear collisions, Annu. Rev. Nucl. Part. Sci. 57, 205 (2007).

[19] M. Strikman and D. Treleani, Measuring Double Parton Distributions in Nucleons at Proton Nucleus Colliders, Phys. Rev. Lett. 88, 031801 (2002).

[20] D. d'Enterria and A. M. Snigirev, Same-sign WW production in proton-nucleus collisions at the LHC as a signal for double parton scattering, Phys. Lett. B 718, 1395 (2013).
[21] S. Salvini, D. Treleani, and G. Calucci, Double parton scatterings in high-energy proton-nucleus collisions and partonic correlations, Phys. Rev. D 89, 016020 (2014).

[22] E. R. Cazaroto, V. P. Gonçalves, and F. S. Navarra, Heavy quark production in $p A$ collisions: The double parton scattering contribution, Mod. Phys. Lett. A 33, 1850141 (2018).

[23] D. d'Enterria and A. Snigirev, Double, triple, and $n$-parton scatterings in high-energy proton and nuclear collisions, Adv. Ser. Dir. High Energy Phys. 29, 159 (2018).

[24] I. Helenius and H. Paukkunen, Double D-meson production in proton-proton and proton-lead collisions at the LHC, Phys. Lett. B 800, 135084 (2020).

[25] B. Blok and F. A. Ceccopieri, Double parton scattering in $p A$ collisions at the LHC revisited, Eur. Phys. J. C 80, 278 (2020).

[26] R. Vogt, Heavy flavor azimuthal correlations in cold nuclear matter, Phys. Rev. C 98, 034907 (2018).

[27] R. Vogt, $b \bar{b}$ kinematic correlations in cold nuclear matter, Phys. Rev. C 101, 024910 (2020).

[28] C. Marquet, C. Roiesnel, and P. Taels, Linearly polarized small- $x$ gluons in forward heavy-quark pair production, Phys. Rev. D 97, 014004 (2018).

[29] J. L. Albacete, G. Giacalone, C. Marquet, and M. Matas, Forward dihadron back-to-back correlations in $p A$ collisions, Phys. Rev. D 99, 014002 (2019).

[30] LHCb Collaboration, $\mathrm{LHCb}$ measurement projections in proton-lead collisions during Run 3 and 4, Report No. LHCb-CONF-2018-005, 2018.

[31] S. Shi, X. Dong, and M. Mustafa, A study of charm quark correlations in ultra-relativistic $p+p$ collisions with PYTHIA, arXiv:1507.00614.

[32] S. Cao, G.-Y. Qin, and S. A. Bass, Modeling of heavy-flavor pair correlations in $\mathrm{Au}-\mathrm{Au}$ collisions at $200 \mathrm{~A} \mathrm{GeV}$ at the BNL relativistic heavy ion collider, Phys. Rev. C 92, 054909 (2015).

[33] X. Zhu, M. Bleicher, S. L. Huang, K. Schweda, H. Stöcker, N. Xu, and P. Zhuang, $D \bar{D}$ correlations as a sensitive probe for thermalization in high-energy nuclear collisions, Phys. Lett. B 647, 366 (2007).

[34] T. Lang, H. van Hees, J. Steinheimer, and M. Bleicher, Dileptons from correlated D- and D-meson decays in the invariant mass range of the QGP thermal radiation using the UrQMD hybrid model, arXiv:1305.7377.

[35] H. He, Y. Liu, and P. Zhuang, $\Omega_{c c c}$ production in high energy nuclear collisions, Phys. Lett. B 746, 59 (2015).

[36] R. J. Fries, B. Müller, C. Nonaka, and S. A. Bass, Hadronization in Heavy Ion Collisions: Recombination and Fragmentation of Partons, Phys. Rev. Lett. 90, 202303 (2003).

[37] R. J. Fries, B. Müller, C. Nonaka, and S. A. Bass, Hadron production in heavy ion collisions: Fragmentation and recombination from a dense parton phase, Phys. Rev. C 68, 044902 (2003).

[38] J.-P. Blaizot, D. De Boni, P. Faccioli, and G. Garberoglio, Heavy quark bound states in a quarkgluon plasma: Dissociation and recombination, Nucl. Phys. A946, 49 (2016).

[39] S. Cho and S. H. Lee, Production of multicharmed hadrons by recombination in heavy ion collisions, Phys. Rev. C 101, 024902 (2020). 
[40] R. L. Thews, M. Schroedter, and J. Rafelski, Enhanced J/ production in deconfined quark matter, Phys. Rev. C 63, 054905 (2001).

[41] A. Andronic, P. Braun-Munzinger, K. Redlich, and J. Stachel, Statistical hadronization of charm in heavy ion collisions at SPS, RHIC and LHC, Phys. Lett. B 571, 36 (2003).

[42] V. Greco, C. M. Ko, and R. Rapp, Quark coalescence for charmed mesons in ultrarelativistic heavy ion collisions, Phys. Lett. B 595, 202 (2004).

[43] B. Abelev et al. (ALICE Collaboration), $J / \psi$ Suppression at Forward Rapidity in $\mathrm{Pb}-\mathrm{Pb}$ Collisions at $\sqrt{s_{N N}}=2.76 \mathrm{TeV}$, Phys. Rev. Lett. 109, 072301 (2012).

[44] X. Yao and B. Mller, Quarkonium inside the quark-gluon plasma: Diffusion, dissociation, recombination, and energy loss, Phys. Rev. D 100, 014008 (2019).

[45] B. Blok, M. Strikman, and U. A. Wiedemann, Hard four-jet production in pA collisions, Eur. Phys. J. C 73, 2433 (2013).

[46] H.-S. Shao, Probing impact-parameter dependent nuclear parton densities from double parton scatterings in heavy-ion collisions, Phys. Rev. D 101, 054036 (2020).

[47] J. M. Link et al. (FOCUS Collaboration), Studies of correlations between $D$ and $\bar{D}$ mesons in high-energy photoproduction, Phys. Lett. B 566, 51 (2003).

[48] E. M. Aitala et al. (E791 Collaboration), Correlations between $D$ and $\bar{D}$ mesons produced in $500 \mathrm{GeV} / c \pi^{-}$nucleon interactions, Eur. Phys. J. Direct 1, 4 (1999).

[49] P. L. Frabetti et al. (E687 Collaboration), Studies of $D \bar{D}$ correlations in high-energy photoproduction, Phys. Lett. B 308, 193 (1993).

[50] S. Barlag et al. (ACCMOR Collaboration), Charmed pair correlations in $\pi^{-} \mathrm{Cu}$ interactions at $230 \mathrm{GeV} / c$, Phys. Lett. B 302, 112 (1993).

[51] R. Aaij et al. (LHCb Collaboration), Observation of double charm production involving open charm in $p p$ collisions at $\sqrt{s}=7 \mathrm{TeV}$, J. High Energy Phys. 06 (2012) 141; 03 (2014) 108(A).

[52] C. Aidala et al. (PHENIX Collaboration), Correlations of $\mu \mu, e \mu$, and $e e$ Pairs in $p+p$ Collisions at $\sqrt{s}=200 \mathrm{GeV}$ and Implications for $c \bar{c}$ and $b \bar{b}$ Production Mechanisms, arXiv:1805.04075 [Phys. Rev. Lett. (to be published)].

[53] C. Albajar et al. (UA1 Collaboration), Measurement of $b \bar{b}$ correlations at the CERN $p \bar{p}$ collider, Z. Phys. C 61, 41 (1994).

[54] F. Abe et al. (CDF Collaboration), Measurement of $b \bar{b}$ rapidity correlations in $p \bar{p}$ collisions at $\sqrt{s}=1.8 \mathrm{TeV}$, Phys. Rev. D 61, 032001 (2000).

[55] D. Acosta et al. (CDF Collaboration), Measurements of $b \bar{b}$ azimuthal production correlations in $p \bar{p}$ collisions at $\sqrt{s}=1.8 \mathrm{TeV}$, Phys. Rev. D 71, 092001 (2005).

[56] T. Aaltonen et al. (CDF Collaboration), Measurement of correlated $b \bar{b}$ production in $p \bar{p}$ collisions at $\sqrt{s}=1960 \mathrm{GeV}$, Phys. Rev. D 77, 072004 (2008).

[57] B. Abbott et al. (D0 Collaboration), The $b \bar{b}$ production cross section and angular correlations in $p \bar{p}$ collisions at $\sqrt{s}=1.8 \mathrm{TeV}$, Phys. Lett. B 487, 264 (2000).

[58] R. Aaij et al. (LHCb Collaboration), Study of $b \bar{b}$ correlations in high energy proton-proton collisions, J. High Energy Phys. 11 (2017) 030.
[59] V. Khachatryan et al. (CMS Collaboration), Measurement of $B \bar{B}$ angular correlations based on secondary vertex reconstruction at $\sqrt{s}=7 \mathrm{TeV}$, J. High Energy Phys. 03 (2011) 136.

[60] R. Aaij et al. (LHCb Collaboration), Observation of $J / \psi$ pair production in $p p$ collisions at $\sqrt{s}=7 \mathrm{TeV}$, Phys. Lett. B 707, 52 (2012).

[61] R. Aaij et al. (LHCb Collaboration), Measurement of the $J / \psi$ pair production cross-section in $p p$ collisions at $\sqrt{s}=13 \mathrm{TeV}$, J. High Energy Phys. 06 (2017) 047; 10 (2017) 068.

[62] R. Aaij et al. (LHCb Collaboration), Production of associated $\Upsilon$ and open charm hadrons in $p p$ collisions at $\sqrt{s}=$ 7 and $8 \mathrm{TeV}$ via double parton scattering, J. High Energy Phys. 07 (2016) 052.

[63] V. Khachatryan et al. (CMS Collaboration), Observation of $\Upsilon(1 \mathrm{~S})$ pair production in proton-proton collisions at $\sqrt{s}=8 \mathrm{TeV}$, J. High Energy Phys. 05 (2017) 013.

[64] V. Khachatryan et al. (CMS Collaboration), Measurement of prompt $J / \psi$ pair production in $p p$ collisions at $\sqrt{s}=7 \mathrm{TeV}$, J. High Energy Phys. 09 (2014) 094.

[65] V. M. Abazov et al. (D0 Collaboration), Evidence for Simultaneous Production of $J / \psi$ and $\Upsilon$ Mesons, Phys. Rev. Lett. 116, 082002 (2016).

[66] F. Abe et al. (CDF Collaboration), Study of four jet events and evidence for double parton interactions in $p \bar{p}$ collisions at $\sqrt{s}=1.8 \mathrm{TeV}$, Phys. Rev. D 47, 4857 (1993).

[67] M. Aaboud et al. (ATLAS Collaboration), Study of hard double-parton scattering in four-jet events in $p p$ collisions at $\sqrt{s}=7 \mathrm{TeV}$ with the ATLAS experiment, J. High Energy Phys. 11 (2016) 110.

[68] F. Abe et al. (CDF Collaboration), Measurement of Double Parton Scattering in $\bar{p} p$ Collisions at $\sqrt{s}=1.8 \mathrm{TeV}$, Phys. Rev. Lett. 79, 584 (1997).

[69] M. Aaboud et al. (ATLAS Collaboration), Measurement of the prompt $J / \psi$ pair production cross-section in $p p$ collisions at $\sqrt{s}=8 \mathrm{TeV}$ with the ATLAS detector, Eur. Phys. J. C 77, 76 (2017).

[70] J.-P. Lansberg, H.-S. Shao, and N. Yamanaka, Indication for double parton scatterings in $W+$ prompt $J / \psi$ production at the LHC, Phys. Lett. B 781, 485 (2018).

[71] J.-P. Lansberg and H.-S. Shao, $J / \psi$-pair production at large momenta: Indications for double parton scatterings and large $\alpha_{s}^{5}$ contributions, Phys. Lett. B 751, 479 (2015).

[72] A. A. Alves Jr. et al. (LHCb Collaboration), The LHCb detector at the LHC, J. Instrum. 3, S08005 (2008).

[73] R. Aaij et al. (LHCb Collaboration), LHCb detector performance, Int. J. Mod. Phys. A 30, 1530022 (2015).

[74] R. Aaij et al., Selection and processing of calibration samples to measure the particle identification performance of the $\mathrm{LHCb}$ experiment in Run 2, Eur. Phys. J. Tech. Instrum. 6, 1 (2018).

[75] F. Archilli et al., Performance of the muon identification at LHCb, J. Instrum. 8, P10020 (2013).

[76] M. Tanabashi et al. (Particle Data Group), Review of particle physics, Phys. Rev. D 98, 030001 (2018), and 2019 update.

[77] R. Aaij et al. (LHCb Collaboration), Prompt and nonprompt $J / \psi$ production and nuclear modification in $p \mathrm{~Pb}$ collisions at $\sqrt{s_{N N}}=8.16 \mathrm{TeV}$, Phys. Lett. B 774, 159 (2017). 
[78] R. Aaij et al. (LHCb Collaboration), Study of prompt $D^{0}$ meson production in $p \mathrm{~Pb}$ at $\sqrt{s_{N N}}=8.16 \mathrm{TeV}$ at $\mathrm{LHCb}$, Report No. LHCb-CONF-2019-004, 2019.

[79] J. P. Alexander et al. (CLEO Collaboration), Absolute Measurement of Hadronic Branching Fractions of the $D_{s}^{+}$ Meson, Phys. Rev. Lett. 100, 161804 (2008).

[80] R. Aaij et al. (LHCb Collaboration), Measurements of prompt charm production cross-sections in $p p$ collisions at $\sqrt{s}=5 \mathrm{TeV}$, J. High Energy Phys. 06 (2017) 147.

[81] T. Skwarnicki, A study of the radiative cascade transitions between the upsilon-prime and upsilon resonances, Ph.D. thesis, Institute of Nuclear Physics, Krakow, 1986, DESYF31-86-02.

[82] See Supplemental Material at http://link.aps.org/supplemental/ 10.1103/PhysRevLett.125.212001 for additional invariant mass distributions and cross-section results.

[83] R. Aaij et al. (LHCb Collaboration), Measurement of $B^{+}, B^{0}$ and $\Lambda_{b}^{0}$ production in $p \mathrm{~Pb}$ collisions at $\sqrt{s_{N N}}=8.16 \mathrm{TeV}$, Phys. Rev. D 99, 052011 (2019).

[84] T. Pierog, Iu. Karpenko, J. M. Katzy, E. Yatsenko, and K. Werner, EPOS LHC: Test of collective hadronization with data measured at the CERN Large Hadron Collider, Phys. Rev. C 92, 034906 (2015).

[85] T. Sjöstrand, S. Mrenna, and P. Skands, A brief introduction to Pythia8.1, Comput. Phys. Commun. 178, 852 (2008).

[86] T. Sjöstrand, S. Mrenna, and P. Skands, Pythia6.4 physics and manual, J. High Energy Phys. 05 (2006) 026.

[87] D. J. Lange, The EvTGEN particle decay simulation package, Nucl. Instrum. Methods Phys. Res., Sect. A 462, 152 (2001).

[88] J. Allison et al. (GEANT4 Collaboration), GEANT4 developments and applications, IEEE Trans. Nucl. Sci. 53 (2006)
270; S. Agostinelli et al. (GEANT4 Collaboration), GEANT4: A simulation toolkit, Nucl. Instrum. Methods Phys. Res., Sect. A 506, 250 (2003).

[89] M. Clemencic, G. Corti, S. Easo, C. R. Jones, S. Miglioranzi, M. Pappagallo, and P. Robbe, The LHCb simulation application, GAUSS: Design, evolution and experience, J. Phys. Conf. Ser. 331, 032023 (2011).

[90] R. Aaij et al. (LHCb Collaboration), Measurement of the track reconstruction efficiency at $\mathrm{LHCb}, \mathrm{J}$. Instrum. 10, P02007 (2015).

[91] M. Pivk and F. R. Le Diberder, sPlot: A statistical tool to unfold data distributions, Nucl. Instrum. Methods Phys. Res., Sect. A 555, 356 (2005).

[92] H.-S. Shao, HELAC-Onia 2.0: An upgraded matrix-element and event generator for heavy quarkonium physics, Comput. Phys. Commun. 198, 238 (2016).

[93] H.-S. Shao, HELAC-Onia: An automatic matrix element generator for heavy quarkonium physics, Comput. Phys. Commun. 184, 2562 (2013).

[94] A. Kusina, J.-P. Lansberg, I. Schienbein, and H.-S. Shao, Gluon Shadowing in Heavy-Flavor Production at the LHC, Phys. Rev. Lett. 121, 052004 (2018).

[95] K. J. Eskola, P. Paakkinen, H. Paukkunen, and C. A. Salgado, EPPS16: Nuclear parton distributions with LHC data, Eur. Phys. J. C 77, 163 (2017).

[96] R. Aaij et al. (LHCb Collaboration), Study of prompt $D^{0}$ meson production in $p \mathrm{~Pb}$ collisions at $\sqrt{s_{N N}}=5 \mathrm{TeV}$, J. High Energy Phys. 10 (2017) 090.

[97] H.-S. Shao, $J / \psi$ meson production in association with an open charm hadron at the LHC: A reappraisal, Phys. Rev. D 102, 034023 (2020).

R. Aaij ${ }^{31}$ C. Abellán Beteta, ${ }^{49}$ T. Ackernley,${ }^{59}$ B. Adeva, ${ }^{45}$ M. Adinolfi,${ }^{53}$ H. Afsharnia, ${ }^{9}$ C. A. Aidala, ${ }^{83}$ S. Aiola, ${ }^{25}$ Z. Ajaltouni, ${ }^{9}$ S. Akar, ${ }^{64}$ J. Albrecht,${ }^{14}$ F. Alessio, ${ }^{47}$ M. Alexander, ${ }^{58}$ A. Alfonso Albero, ${ }^{44}$ Z. Aliouche,${ }^{61}$ G. Alkhazov,${ }^{37}$ P. Alvarez Cartelle, ${ }^{47}$ A. A. Alves Jr., ${ }^{45}$ S. Amato, ${ }^{2}$ Y. Amhis,${ }^{11}$ L. An, ${ }^{21}$ L. Anderlini, ${ }^{21}$ G. Andreassi,${ }^{48}$ A. Andreianov,${ }^{37}$ M. Andreotti, ${ }^{20}$ F. Archilli, ${ }^{16}$ A. Artamonov, ${ }^{43}$ M. Artuso,${ }^{67}$ K. Arzymatov, ${ }^{41}$ E. Aslanides,${ }^{10}$ M. Atzeni, ${ }^{49}$ B. Audurier, ${ }^{11}$ S. Bachmann, ${ }^{16}$ M. Bachmayer, ${ }^{48}$ J. J. Back, ${ }^{55}$ S. Baker, ${ }^{60}$ P. Baladron Rodriguez, ${ }^{45}$ V. Balagura, ${ }^{11, a}$ W. Baldini, ${ }^{20}$

J. Baptista Leite, ${ }^{1}$ R. J. Barlow, ${ }^{61}$ S. Barsuk,${ }^{11}$ W. Barter, ${ }^{60}$ M. Bartolini, ${ }^{23,47, b}$ F. Baryshnikov, ${ }^{80}$ J. M. Basels, ${ }^{13}$ G. Bassi, ${ }^{28}$ V. Batozskaya, ${ }^{35}$ B. Batsukh,${ }^{67}$ A. Battig, ${ }^{14}$ A. Bay, ${ }^{48}$ M. Becker,${ }^{14}$ F. Bedeschi, ${ }^{28}$ I. Bediaga, ${ }^{1}$ A. Beiter, ${ }^{67}$ V. Belavin,${ }^{41}$ S. Belin, ${ }^{26}$ V. Bellee, ${ }^{48}$ K. Belous,${ }^{43}$ I. Belov, ${ }^{39}$ I. Belyaev, ${ }^{38}$ G. Bencivenni, ${ }^{22}$ E. Ben-Haim, ${ }^{12}$ A. Berezhnoy, ${ }^{39}$ R. Bernet, ${ }^{49}$ D. Berninghoff, ${ }^{16}$ H. C. Bernstein, ${ }^{67}$ C. Bertella, ${ }^{47}$ E. Bertholet, ${ }^{12}$ A. Bertolin, ${ }^{27}$ C. Betancourt, ${ }^{49}$ F. Betti,,${ }^{19, c}$ M. O. Bettler, ${ }^{54}$ Ia. Bezshyiko, ${ }^{49}$ S. Bhasin, ${ }^{53}$ J. Bhom, ${ }^{33}$ L. Bian, ${ }^{72}$ M. S. Bieker, ${ }^{14}$ S. Bifani, ${ }^{52}$ P. Billoir, ${ }^{12}$ M. Birch, ${ }^{60}$ F. C. R. Bishop, ${ }^{54}$ A. Bizzeti, ${ }^{21, \mathrm{~d}}$ M. Bjørn, ${ }^{62}$ M. P. Blago,${ }^{47}$ T. Blake,${ }^{55}$ F. Blanc, ${ }^{48}$ S. Blusk,${ }^{67}$ D. Bobulska,${ }^{58}$ V. Bocci,${ }^{30}$ J. A. Boelhauve, ${ }^{14}$ O. Boente Garcia, ${ }^{45}$ T. Boettcher, ${ }^{63}$ A. Boldyrev, ${ }^{81}$ A. Bondar, ${ }^{42, e}$ N. Bondar, ${ }^{37,47}$ S. Borghi, ${ }^{61}$ M. Borisyak,,${ }^{41}$ M. Borsato, ${ }^{16}$ J. T. Borsuk, ${ }^{33}$ S. A. Bouchiba, ${ }^{48}$ T. J. V. Bowcock, ${ }^{59}$ A. Boyer, ${ }^{47}$ C. Bozzi,${ }^{20}$ M. J. Bradley, ${ }^{60}$ S. Braun, ${ }^{65}$

A. Brea Rodriguez,${ }^{45}$ M. Brodski, ${ }^{47}$ J. Brodzicka, ${ }^{33}$ A. Brossa Gonzalo, ${ }^{55}$ D. Brundu, ${ }^{26}$ E. Buchanan, ${ }^{53}$ A. Buonaura ${ }^{49}$ C. Burr, ${ }^{47}$ A. Bursche, ${ }^{26}$ A. Butkevich, ${ }^{40}$ J. S. Butter, ${ }^{31}$ J. Buytaert, ${ }^{47}$ W. Byczynski, ${ }^{47}$ S. Cadeddu, ${ }^{26}$ H. Cai,${ }^{72}$ R. Calabrese, ${ }^{20, f}$ L. Calefice, ${ }^{14}$ L. Calero Diaz, ${ }^{22}$ S. Cali, ${ }^{22}$ R. Calladine, ${ }^{52}$ M. Calvi, ${ }^{24, g}$ M. Calvo Gomez, ${ }^{44, h}$ P. Camargo Magalhaes, ${ }^{53}$ A. Camboni, ${ }^{44}$ P. Campana, ${ }^{22}$ D. H. Campora Perez ${ }^{47}$ A. F. Campoverde Quezada, ${ }^{5}$ S. Capelli, ${ }^{24, g}$ L. Capriotti, ${ }^{19, c}$ A. Carbone, ${ }^{19, \mathrm{c}}$ G. Carboni, ${ }^{29}$ R. Cardinale, ${ }^{23, \mathrm{~b}}$ A. Cardini, ${ }^{26}$ I. Carli, ${ }^{6}$ P. Carniti, ${ }^{24, \mathrm{~g}}$ K. Carvalho Akiba, ${ }^{31}$ A. Casais Vidal, ${ }^{45}$ G. Casse,${ }^{59}$ M. Cattaneo, ${ }^{47}$ G. Cavallero, ${ }^{47}$ S. Celani, ${ }^{48}$ R. Cenci, ${ }^{28}$ J. Cerasoli, ${ }^{10}$ A. J. Chadwick, ${ }^{59}$ M. G. Chapman, ${ }^{53}$ M. Charles, ${ }^{12}$ Ph. Charpentier, ${ }^{47}$ G. Chatzikonstantinidis, ${ }^{52}$ C. A. Chavez Barajas, ${ }^{59}$ M. Chefdeville, ${ }^{8}$ 
C. Chen, ${ }^{3}$ S. Chen, ${ }^{26}$ A. Chernov, ${ }^{33}$ S.-G. Chitic, ${ }^{47}$ V. Chobanova, ${ }^{45}$ S. Cholak, ${ }^{48}$ M. Chrzaszcz, ${ }^{33}$ A. Chubykin,${ }^{37}$ V. Chulikov, ${ }^{37}$ P. Ciambrone, ${ }^{22}$ M. F. Cicala, ${ }^{55}$ X. Cid Vidal, ${ }^{45}$ G. Ciezarek, ${ }^{47}$ P. E. L. Clarke,${ }^{57}$ M. Clemencic,${ }^{47}$ H. V. Cliff,${ }^{54}$ J. Closier, ${ }^{47}$ J. L. Cobbledick, ${ }^{61}$ V. Coco,${ }^{47}$ J. A. B. Coelho, ${ }^{11}$ J. Cogan, ${ }^{10}$ E. Cogneras, ${ }^{9}$ L. Cojocariu, ${ }^{36}$ P. Collins, ${ }^{47}$ T. Colombo, ${ }^{47}$ L. Congedo, ${ }^{18}$ A. Contu, ${ }^{26}$ N. Cooke, ${ }^{52}$ G. Coombs,${ }^{58}$ S. Coquereau, ${ }^{44}$ G. Corti, ${ }^{47}$ C. M. Costa Sobral,${ }^{55}$ B. Couturier, ${ }^{47}$ D. C. Craik, ${ }^{63}$ J. Crkovská, ${ }^{66}$ M. Cruz Torres,${ }^{1, i}$ R. Currie, ${ }^{57}$ C. L. Da Silva, ${ }^{66}$ E. Dall'Occo, ${ }^{14}$ J. Dalseno, ${ }^{45}$ C. D'Ambrosio, ${ }^{47}$ A. Danilina, ${ }^{38}$ P. d'Argent, ${ }^{47}$ A. Davis,${ }^{61}$ O. De Aguiar Francisco, ${ }^{47}$ K. De Bruyn, ${ }^{47}$ S. De Capua,${ }^{61}$ M. De Cian, ${ }^{48}$ J. M. De Miranda, ${ }^{1}$ L. De Paula, ${ }^{2}$ M. De Serio, ${ }^{18, j}$ D. De Simone, ${ }^{49}$ P. De Simone, ${ }^{22}$ J. A. de Vries, ${ }^{78}$ C. T. Dean, ${ }^{66}$ W. Dean, ${ }^{83}$ D. Decamp,${ }^{8}$ L. Del Buono, ${ }^{12}$ B. Delaney ${ }^{54}$ H.-P. Dembinski, ${ }^{14}$ A. Dendek,${ }^{34}$ V. Denysenko, ${ }^{49}$ D. Derkach, ${ }^{81}$ O. Deschamps, ${ }^{9}$ F. Desse, ${ }^{11}$ F. Dettori, ${ }^{26, k}$ B. Dey, ${ }^{7}$ A. Di Canto,${ }^{47}$ P. Di Nezza, ${ }^{22}$ S. Didenko, ${ }^{80}$ L. Dieste Maronas, ${ }^{45}$ H. Dijkstra, ${ }^{47}$ V. Dobishuk, ${ }^{51}$ A. M. Donohoe, ${ }^{17}$ F. Dordei, ${ }^{26}$ M. Dorigo, ${ }^{28,1}$ A. C. dos Reis, ${ }^{1}$ L. Douglas, ${ }^{58}$ A. Dovbnya, ${ }^{50}$ A. G. Downes, ${ }^{8}$ K. Dreimanis, ${ }^{59}$ M. W. Dudek, ${ }^{33}$ L. Dufour,${ }^{47}$ V. Duk, ${ }^{76}$ P. Durante, ${ }^{47}$ J. M. Durham, ${ }^{66}$ D. Dutta, ${ }^{61}$ M. Dziewiecki, ${ }^{16}$ A. Dziurda, ${ }^{33}$ A. Dzyuba, ${ }^{37}$ S. Easo,${ }^{56}$ U. Egede, ${ }^{69}$ V. Egorychev, ${ }^{38}$ S. Eidelman, ${ }^{42, e}$ S. Eisenhardt ${ }^{57}$ S. Ek-In, ${ }^{48}$ L. Eklund ${ }^{58}$ S. Ely, ${ }^{67}$ A. Ene, ${ }^{36}$ E. Epple, ${ }^{66}$ S. Escher, ${ }^{13}$ J. Eschle, ${ }^{49}$ S. Esen,${ }^{31}$ T. Evans, ${ }^{47}$ A. Falabella, ${ }^{19}$ J. Fan, ${ }^{3}$ Y. Fan, ${ }^{5}$ B. Fang, ${ }^{72}$ N. Farley, ${ }^{52}$ S. Farry,${ }^{59}$ D. Fazzini, ${ }^{11}$ P. Fedin, ${ }^{38}$ M. Féo, ${ }^{47}$ P. Fernandez Declara, ${ }^{47}$ A. Fernandez Prieto, ${ }^{45}$ J. M. Fernandez-tenllado Arribas,${ }^{44}$ F. Ferrari, ${ }^{19, \mathrm{c}}$ L. Ferreira Lopes ${ }^{48}$ F. Ferreira Rodrigues, ${ }^{2}$ S. Ferreres Sole,${ }^{31}$ M. Ferrillo, ${ }^{49}$ M. Ferro-Luzzi, ${ }^{47}$ S. Filippov, ${ }^{40}$ R. A. Fini, ${ }^{18}$ M. Fiorini, ${ }^{20, f}$ M. Firlej, ${ }^{34}$ K. M. Fischer, ${ }^{62}$ C. Fitzpatrick, ${ }^{61}$ T. Fiutowski, ${ }^{34}$ F. Fleuret, ${ }^{11, a}$ M. Fontana, ${ }^{47}$ F. Fontanelli, ${ }^{23, b}$ R. Forty, ${ }^{47}$ V. Franco Lima, ${ }^{59}$ M. Franco Sevilla, ${ }^{65}$ M. Frank, ${ }^{47}$ E. Franzoso, ${ }^{20}$ G. Frau, ${ }^{16}$ C. Frei, ${ }^{47}$ D. A. Friday, ${ }^{58}$ J. Fu, ${ }^{25, m}$ Q. Fuehring, ${ }^{14}$ W. Funk,${ }^{47}$ E. Gabriel,${ }^{31}$ T. Gaintseva, ${ }^{41}$ A. Gallas Torreira,${ }^{45}$ D. Galli, ${ }^{19, c}$ S. Gallorini, ${ }^{27}$ S. Gambetta, ${ }^{57}$ Y. Gan, ${ }^{3}$ M. Gandelman, ${ }^{2}$ P. Gandini, ${ }^{25}$ Y. Gao, ${ }^{4}$ M. Garau, ${ }^{26}$ L. M. Garcia Martin, ${ }^{46}$ P. Garcia Moreno, ${ }^{44}$

J. García Pardiñas, ${ }^{49}$ B. Garcia Plana, ${ }^{45}$ F. A. Garcia Rosales, ${ }^{11}$ L. Garrido, ${ }^{44}$ D. Gascon, ${ }^{44}$ C. Gaspar, ${ }^{47}$ R. E. Geertsema,${ }^{31}$ D. Gerick, ${ }^{16}$ L. L. Gerken, ${ }^{14}$ E. Gersabeck, ${ }^{61}$ M. Gersabeck, ${ }^{61}$ T. Gershon, ${ }^{55}$ D. Gerstel, ${ }^{10}$ Ph. Ghez, ${ }^{8}$ V. Gibson, ${ }^{54}$ M. Giovannetti, ${ }^{2, n}$ A. Gioventù ${ }^{45}$ P. Gironella Gironell, ${ }^{44}$ L. Giubega,${ }^{36}$ C. Giugliano, ${ }^{20, f}$ K. Gizdov, ${ }^{57}$

E. L. Gkougkousis, ${ }^{47}$ V. V. Gligorov,${ }^{12}$ C. Göbel, ${ }^{70}$ E. Golobardes,${ }^{44, h}$ D. Golubkov, ${ }^{38}$ A. Golutvin, ${ }^{60,80}$ A. Gomes, ${ }^{1, o}$ S. Gomez Fernandez, ${ }^{44}$ F. Goncalves Abrantes, ${ }^{70}$ M. Goncerz, ${ }^{33}$ G. Gong, ${ }^{3}$ P. Gorbounov, ${ }^{38}$ I. V. Gorelov, ${ }^{39}$ C. Gotti, ${ }^{24, g}$ E. Govorkova, ${ }^{31}$ J. P. Grabowski, ${ }^{16}$ R. Graciani Diaz, ${ }^{44}$ T. Grammatico, ${ }^{12}$ L. A. Granado Cardoso, ${ }^{47}$ E. Graugés ${ }^{44}$ E. Graverini, ${ }^{48}$ G. Graziani, ${ }^{21}$ A. Grecu, ${ }^{36}$ L. M. Greeven, ${ }^{31}$ P. Griffith, ${ }^{20, f}$ L. Grillo, ${ }^{61}$ S. Gromov, ${ }^{80}$ L. Gruber,${ }^{47}$ B. R. Gruberg Cazon, ${ }^{62}$ C. Gu, ${ }^{3}$ M. Guarise, ${ }^{20}$ P. A. Günther, ${ }^{16}$ E. Gushchin,${ }^{40}$ A. Guth, ${ }^{13}$ Y. Guz, ${ }^{43,47}$ T. Gys, ${ }^{47}$ T. Hadavizadeh, ${ }^{69}$ G. Haefeli, ${ }^{48}$ C. Haen, ${ }^{47}$ J. Haimberger, ${ }^{47}$ S. C. Haines,${ }^{54}$ T. Halewood-leagas, ${ }^{59}$ P. M. Hamilton, ${ }^{65}$ Q. Han, ${ }^{7}$ X. Han, ${ }^{16}$ T. H. Hancock,${ }^{62}$ S. Hansmann-Menzemer, ${ }^{16}$ N. Harnew,${ }^{62}$ T. Harrison,,${ }^{59}$ R. Hart, ${ }^{31}$ C. Hasse, ${ }^{47}$ M. Hatch, ${ }^{47}$ J. He,${ }^{5}$ M. Hecker, ${ }^{60}$ K. Heijhoff, ${ }^{31}$ K. Heinicke, ${ }^{14}$ A. M. Hennequin, ${ }^{47}$ K. Hennessy,${ }^{59}$ L. Henry, ${ }^{25,46}$ J. Heuel, ${ }^{13}$ A. Hicheur, ${ }^{68}$ D. Hill, ${ }^{62}$ M. Hilton, ${ }^{61}$ S. E. Hollitt, ${ }^{14}$ P. H. Hopchev, ${ }^{48}$ J. Hu, ${ }^{16}$ J. Hu, ${ }^{71}$ W. Hu, W. Huang, ${ }^{5}$ X. Huang,${ }^{72}$ W. Hulsbergen, ${ }^{31}$ T. Humair, ${ }^{60}$ R. J. Hunter, ${ }^{55}$ M. Hushchyn, ${ }^{81}$ D. Hutchcroft, ${ }^{59}$ D. Hynds, ${ }^{31}$ P. Ibis, ${ }^{14}$ M. Idzik, ${ }^{34}$ D. Ilin, ${ }^{37}$ P. Ilten, ${ }^{52}$ A. Inglessi, ${ }^{37}$ A. Ishteev, ${ }^{80}$ K. Ivshin, ${ }^{37}$ R. Jacobsson, ${ }^{47}$ S. Jakobsen, ${ }^{47}$ E. Jans,${ }^{31}$ B. K. Jashal,${ }^{46}$ A. Jawahery, ${ }^{65}$ V. Jevtic, ${ }^{14}$ M. Jezabek, ${ }^{33}$ F. Jiang, ${ }^{3}$ M. John, ${ }^{62}$ D. Johnson, ${ }^{47}$ C. R. Jones,${ }^{54}$ T. P. Jones,${ }^{55}$ B. Jost, ${ }^{47}$ N. Jurik, ${ }^{62}$ S. Kandybei, ${ }^{50}$ Y. Kang, ${ }^{3}$ M. Karacson, ${ }^{47}$ J. M. Kariuki, ${ }^{53}$ N. Kazeev, ${ }^{81}$ M. Kecke, ${ }^{16}$ F. Keizer,${ }^{54,47}$ M. Kelsey, ${ }^{67}$ M. Kenzie, ${ }^{55}$ T. Ketel ${ }^{32}$ B. Khanji, ${ }^{47}$ A. Kharisova, ${ }^{82}$ S. Kholodenko, ${ }^{43}$ K. E. Kim, ${ }^{67}$ T. Kirn, ${ }^{13}$ V. S. Kirsebom, ${ }^{48}$ O. Kitouni, ${ }^{63}$ S. Klaver ${ }^{22}$

K. Klimaszewski, ${ }^{35}$ S. Koliiev, ${ }^{51}$ A. Kondybayeva, ${ }^{80}$ A. Konoplyannikov ${ }^{38}$ P. Kopciewicz, ${ }^{34}$ R. Kopecna, ${ }^{16}$

P. Koppenburg, ${ }^{31}$ M. Korolev, ${ }^{39}$ I. Kostiuk, ${ }^{31,51}$ O. Kot, ${ }^{51}$ S. Kotriakhova, ${ }^{37,30}$ P. Kravchenko, ${ }^{37}$ L. Kravchuk, ${ }^{40}$ R. D. Krawczyk,${ }^{47}$ M. Kreps, ${ }^{55}$ F. Kress, ${ }^{60}$ S. Kretzschmar, ${ }^{13}$ P. Krokovny, ${ }^{42, \mathrm{e}}$ W. Krupa ${ }^{34}$ W. Krzemien, ${ }^{35}$ W. Kucewicz, ${ }^{33, p}$ M. Kucharczyk, ${ }^{33}$ V. Kudryavtsev, ${ }^{42, e}$ H. S. Kuindersma, ${ }^{31}$ G. J. Kunde, ${ }^{66}$ T. Kvaratskheliya, ${ }^{38}$ D. Lacarrere, ${ }^{47}$ G. Lafferty, ${ }^{61}$ A. Lai, ${ }^{26}$ A. Lampis, ${ }^{26}$ D. Lancierini ${ }^{49}$ J. J. Lane,${ }^{61}$ R. Lane, ${ }^{53}$ G. Lanfranchi ${ }^{22}$ C. Langenbruch, ${ }^{13}$ J. Langer ${ }^{14}$ O. Lantwin ${ }^{49,80}$ T. Latham, ${ }^{55}$ F. Lazzari, ${ }^{28, q}$ R. Le Gac, ${ }^{10}$ S. H. Lee, ${ }^{83}$ R. Lefèvre, ${ }^{9}$ A. Leflat, ${ }^{39,47}$ S. Legotin, ${ }^{80}$ O. Leroy, ${ }^{10}$ T. Lesiak, ${ }^{33}$ B. Leverington, ${ }^{16}$ H. Li ${ }^{71}$ L. Li ${ }^{62}$ P. Li ${ }^{16}$ X. Li, ${ }^{66}$ Y. Li, ${ }^{6}$ Y. Li,${ }^{6}$ Z. Li ${ }^{67}$ X. Liang, ${ }^{67}$ T. Lin,${ }^{60}$ R. Lindner, ${ }^{47}$ V. Lisovskyi, ${ }^{14}$ R. Litvinov, ${ }^{26}$ G. Liu, ${ }^{71}$ H. Liu, ${ }^{5}$ S. Liu, ${ }^{6}$ X. Liu, ${ }^{3}$ A. Loi, ${ }^{26}$ J. Lomba Castro, ${ }^{45}$ I. Longstaff, ${ }^{58}$ J. H. Lopes, ${ }^{2}$ G. Loustau, ${ }^{49}$ G. H. Lovell, ${ }^{54}$ Y. Lu, ${ }^{6}$ D. Lucchesi,${ }^{27, r}$ S. Luchuk,${ }^{40}$ M. Lucio Martinez ${ }^{31}$ V. Lukashenko, ${ }^{31}$ Y. Luo, ${ }^{3}$ A. Lupato, ${ }^{61}$ E. Luppi,${ }^{20, f}$ O. Lupton, ${ }^{55}$ A. Lusiani,${ }^{28, s}$ X. Lyu,${ }^{5}$ L. Ma, ${ }^{6}$ S. Maccolini, ${ }^{19, c}$ F. Machefert, ${ }^{11}$ F. Maciuc, ${ }^{36}$ V. Macko, ${ }^{48}$ P. Mackowiak, ${ }^{14}$ S. Maddrell-Mander,${ }^{53}$ O. Madejczyk, ${ }^{34}$ L. R. Madhan Mohan,${ }^{53}$ O. Maev, ${ }^{37}$ A. Maevskiy,${ }^{81}$ D. Maisuzenko, ${ }^{37}$ M. W. Majewski, ${ }^{34}$ S. Malde,${ }^{62}$ B. Malecki, ${ }^{47}$ A. Malinin, ${ }^{79}$ T. Maltsev, ${ }^{42, e}$ H. Malygina, ${ }^{16}$ G. Manca, ${ }^{26, k}$ 
G. Mancinelli, ${ }^{10}$ R. Manera Escalero, ${ }^{44}$ D. Manuzzi,${ }^{19, c}$ D. Marangotto, ${ }^{25, m}$ J. Maratas,,${ }^{9, t}$ J. F. Marchand, ${ }^{8}$ U. Marconi, ${ }^{19}$ S. Mariani, ${ }^{21,47, u}$ C. Marin Benito, ${ }^{11}$ M. Marinangeli, ${ }^{48}$ P. Marino, ${ }^{48}$ J. Marks, ${ }^{16}$ P. J. Marshall, ${ }^{59}$ G. Martellotti, ${ }^{30}$ L. Martinazzoli, ${ }^{47}$ M. Martinelli, ${ }^{24, g}$ D. Martinez Santos, ${ }^{45}$ F. Martinez Vidal,${ }^{46}$ A. Massafferri, ${ }^{1}$ M. Materok, ${ }^{13}$ R. Matev, ${ }^{47}$ A. Mathad ${ }^{49}$ Z. Mathe, ${ }^{47}$ V. Matiunin, ${ }^{38}$ C. Matteuzzi, ${ }^{24}$ K. R. Mattioli, ${ }^{83}$ A. Mauri, ${ }^{49}$ E. Maurice, ${ }^{11, a}$ J. Mauricio, ${ }^{44}$ M. Mazurek, ${ }^{35}$ M. McCann,${ }^{60}$ L. Mcconnell, ${ }^{17}$ T. H. Mcgrath, ${ }^{61}$ A. McNab,${ }^{61}$ R. McNulty, ${ }^{17}$ J. V. Mead, ${ }^{59}$ B. Meadows,${ }^{64}$ C. Meaux,${ }^{10}$ G. Meier ${ }^{14}$ N. Meinert, ${ }^{75}$ D. Melnychuk,${ }^{35}$ S. Meloni,${ }^{24, g}$ M. Merk ${ }^{31,78}$ A. Merli, ${ }^{25}$ L. Meyer Garcia, ${ }^{2}$ M. Mikhasenko, ${ }^{47}$ D. A. Milanes, ${ }^{73}$ E. Millard ${ }^{55}$ M. Milovanovic,${ }^{47}$ M.-N. Minard, ${ }^{8}$ L. Minzoni, ${ }^{20, f}$ S. E. Mitchell,${ }^{57}$ B. Mitreska, ${ }^{61}$ D. S. Mitzel, ${ }^{47}$ A. Mödden, ${ }^{14}$ R. A. Mohammed, ${ }^{62}$ R. D. Moise,${ }^{60}$ T. Mombächer, ${ }^{14}$ I. A. Monroy, ${ }^{73}$ S. Monteil,,${ }^{9}$ M. Morandin, ${ }^{27}$ G. Morello, ${ }^{22}$ M. J. Morello, ${ }^{28, s}$ J. Moron, ${ }^{34}$ A. B. Morris, ${ }^{74}$ A. G. Morris, ${ }^{55}$ R. Mountain, ${ }^{67}$ H. Mu, ${ }^{3}$ F. Muheim, ${ }^{57}$ M. Mukherjee, ${ }^{7}$ M. Mulder, ${ }^{47}$ D. Müller, ${ }^{47}$ K. Müller, ${ }^{49}$ C. H. Murphy, ${ }^{62}$ D. Murray, ${ }^{61}$ P. Muzzetto, ${ }^{26}$ P. Naik, ${ }^{53}$ T. Nakada, ${ }^{48}$ R. Nandakumar, ${ }^{56}$ T. Nanut, ${ }^{48}$ I. Nasteva, ${ }^{2}$ M. Needham, ${ }^{57}$ I. Neri, ${ }^{20, f}$ N. Neri, ${ }^{25, m}$ S. Neubert, ${ }^{74}$ N. Neufeld, ${ }^{47}$ R. Newcombe, ${ }^{60}$ T. D. Nguyen,${ }^{48}$ C. Nguyen-Mau, ${ }^{48, v}$ E. M. Niel,${ }^{11}$ S. Nieswand, ${ }^{13}$ N. Nikitin, ${ }^{39}$ N. S. Nolte, ${ }^{47}$ C. Nunez, ${ }^{83}$ A. Oblakowska-Mucha, ${ }^{34}$ V. Obraztsov, ${ }^{43}$ S. Ogilvy, ${ }^{58}$ D. P. O'Hanlon, ${ }^{53}$ R. Oldeman, ${ }^{26, k}$ C. J. G. Onderwater, ${ }^{77}$ J. D. Osborn, ${ }^{83}$ A. Ossowska, ${ }^{33}$ J. M. Otalora Goicochea, ${ }^{2}$ T. Ovsiannikova, ${ }^{38}$ P. Owen, ${ }^{49}$ A. Oyanguren, ${ }^{46}$ B. Pagare, ${ }^{55}$

P. R. Pais, ${ }^{47}$ T. Pajero, ${ }^{28,47, s}$ A. Palano, ${ }^{18}$ M. Palutan, ${ }^{22}$ Y. Pan,${ }^{61}$ G. Panshin,${ }^{82}$ A. Papanestis, ${ }^{56}$ M. Pappagallo, ${ }^{57}$ L. L. Pappalardo, ${ }^{20, f}$ C. Pappenheimer, ${ }^{64}$ W. Parker, ${ }^{65}$ C. Parkes,${ }^{61}$ C. J. Parkinson, ${ }^{45}$ B. Passalacqua ${ }^{20}$ G. Passaleva, ${ }^{21,47}$ A. Pastore, ${ }^{18}$ M. Patel,${ }^{60}$ C. Patrignani, ${ }^{19, c}$ C. J. Pawley, ${ }^{78}$ A. Pearce, ${ }^{47}$ A. Pellegrino, ${ }^{31}$ M. Pepe Altarelli, ${ }^{47}$ S. Perazzini, ${ }^{19}$ D. Pereima, ${ }^{38}$ P. Perret, ${ }^{9}$ K. Petridis,${ }^{53}$ A. Petrolini, ${ }^{23, b}$ A. Petrov,${ }^{79}$ S. Petrucci, ${ }^{57}$ M. Petruzzo, ${ }^{25}$ A. Philippov, ${ }^{41}$ L. Pica,${ }^{28}$ M. Piccini ${ }^{76}$ B. Pietrzyk,${ }^{8}$ G. Pietrzyk ${ }^{48}$ M. Pili, ${ }^{62}$ D. Pinci,${ }^{30}$ J. Pinzino, ${ }^{47}$ F. Pisani,${ }^{47}$ A. Piucci,${ }^{16}$ Resmi P. K, ${ }^{10}$ V. Placinta, ${ }^{36}$ S. Playfer, ${ }^{57}$ J. Plews, ${ }^{52}$ M. Plo Casasus, ${ }^{45}$ F. Polci, ${ }^{12}$ M. Poli Lener, ${ }^{22}$ M. Poliakova, ${ }^{67}$ A. Poluektov, ${ }^{10}$ N. Polukhina, ${ }^{80, w}$ I. Polyakov, ${ }^{67}$ E. Polycarpo, ${ }^{2}$ G. J. Pomery, ${ }^{53}$ S. Ponce, ${ }^{47}$ A. Popov, ${ }^{43}$ D. Popov, ${ }^{5,47}$ S. Popov, ${ }^{41}$ S. Poslavskii, ${ }^{43}$ K. Prasanth, ${ }^{33}$ L. Promberger, ${ }^{47}$ C. Prouve, ${ }^{45}$ V. Pugatch, ${ }^{51}$ A. Puig Navarro, ${ }^{49}$ H. Pullen, ${ }^{62}$ G. Punzi,${ }^{28, x}$ W. Qian, ${ }^{5}$ J. Qin, ${ }^{5}$ R. Quagliani, ${ }^{12}$ B. Quintana, ${ }^{8}$ N. V. Raab ${ }^{17}$ R. I. Rabadan Trejo, ${ }^{10}$ B. Rachwal, ${ }^{34}$ J. H. Rademacker, ${ }^{53}$ M. Rama, ${ }^{28}$ M. Ramos Pernas, ${ }^{45}$ M. S. Rangel, ${ }^{2}$ F. Ratnikov, ${ }^{41,81}$ G. Raven, ${ }^{32}$ M. Reboud, ${ }^{8}$ F. Redi, ${ }^{48}$ F. Reiss, ${ }^{12}$ C. Remon Alepuz,${ }^{46}$ Z. Ren, ${ }^{3}$ V. Renaudin, ${ }^{62}$ R. Ribatti, ${ }^{28}$ S. Ricciardi, ${ }^{56}$ D. S. Richards,${ }^{56}$ K. Rinnert, ${ }^{59}$ P. Robbe, ${ }^{11}$ A. Robert, ${ }^{12}$ G. Robertson, ${ }^{57}$ A. B. Rodrigues, ${ }^{48}$ E. Rodrigues, ${ }^{59}$ J. A. Rodriguez Lopez ${ }^{73}$ M. Roehrken, ${ }^{47}$ A. Rollings,${ }^{62}$ P. Roloff, ${ }^{47}$ V. Romanovskiy, ${ }^{43}$ M. Romero Lamas, ${ }^{45}$ A. Romero Vidal, ${ }^{45}$ J. D. Roth, ${ }^{83}$ M. Rotondo, ${ }^{22}$ M. S. Rudolph ${ }^{67}$ T. Ruf, ${ }^{47}$ J. Ruiz Vidal, ${ }^{46}$ A. Ryzhikov, ${ }^{81}$ J. Ryzka, ${ }^{34}$ J. J. Saborido Silva, ${ }^{45}$ N. Sagidova, ${ }^{37}$ N. Sahoo, ${ }^{55}$ B. Saitta, ${ }^{26, k}$

D. Sanchez Gonzalo, ${ }^{44}$ C. Sanchez Gras, ${ }^{31}$ C. Sanchez Mayordomo, ${ }^{46}$ R. Santacesaria, ${ }^{30}$ C. Santamarina Rios, ${ }^{45}$ M. Santimaria, ${ }^{22}$ E. Santovetti, ${ }^{29, \mathrm{n}}$ D. Saranin,${ }^{80}$ G. Sarpis, ${ }^{61}$ M. Sarpis, ${ }^{74}$ A. Sarti, ${ }^{30}$ C. Satriano, ${ }^{30, y}$ A. Satta, ${ }^{29}$ M. Saur, ${ }^{5}$ D. Savrina, ${ }^{38,39}$ H. Sazak, ${ }^{9}$ L. G. Scantlebury Smead, ${ }^{62}$ S. Schael,${ }^{13}$ M. Schellenberg, ${ }^{14}$ M. Schiller, ${ }^{58}$ H. Schindler ${ }^{47}$

M. Schmelling, ${ }^{15}$ T. Schmelzer, ${ }^{14}$ B. Schmidt, ${ }^{47}$ O. Schneider, ${ }^{48}$ A. Schopper, ${ }^{47}$ H. F. Schreiner, ${ }^{64}$ M. Schubiger, ${ }^{31}$

S. Schulte, ${ }^{48}$ M. H. Schune, ${ }^{11}$ R. Schwemmer, ${ }^{47}$ B. Sciascia, ${ }^{22}$ A. Sciubba, ${ }^{30}$ S. Sellam, ${ }^{68}$ A. Semennikov, ${ }^{38}$

M. Senghi Soares, ${ }^{32}$ A. Sergi, ${ }^{52,47}$ N. Serra, ${ }^{49}$ J. Serrano, ${ }^{10}$ L. Sestini, ${ }^{27}$ A. Seuthe, ${ }^{14}$ P. Seyfert, ${ }^{47}$ D. M. Shangase, ${ }^{83}$ M. Shapkin, ${ }^{43}$ I. Shchemerov,${ }^{80}$ L. Shchutska, ${ }^{48}$ T. Shears, ${ }^{59}$ L. Shekhtman, ${ }^{42, \mathrm{e}}$ Z. Shen, ${ }^{4}$ V. Shevchenko, ${ }^{79}$ E. B. Shields,${ }^{24, g}$ E. Shmanin, ${ }^{80}$ J. D. Shupperd, ${ }^{67}$ B. G. Siddi, ${ }^{20}$ R. Silva Coutinho, ${ }^{49}$ L. Silva de Oliveira, ${ }^{2}$ G. Simi, ${ }^{27}$ S. Simone, ${ }^{18, j}$ I. Skiba, ${ }^{20, \mathrm{f}}$ N. Skidmore, ${ }^{74}$ T. Skwarnicki, ${ }^{67}$ M. W. Slater, ${ }^{52}$ J. C. Smallwood, ${ }^{62}$ J. G. Smeaton, ${ }^{54}$ A. Smetkina, ${ }^{38}$ E. Smith, ${ }^{13}$ M. Smith, ${ }^{60}$ A. Snoch, ${ }^{31}$ M. Soares, ${ }^{19}$ L. Soares Lavra, ${ }^{9}$ M. D. Sokoloff, ${ }^{64}$ F. J. P. Soler, ${ }^{58}$ A. Solovev, ${ }^{37}$ I. Solovyev, ${ }^{37}$ F. L. Souza De Almeida, ${ }^{2}$ B. Souza De Paula, ${ }^{2}$ B. Spaan, ${ }^{14}$ E. Spadaro Norella, ${ }^{25, m}$ P. Spradlin,${ }^{58}$ F. Stagni, ${ }^{47}$ M. Stahl, ${ }^{64}$ S. Stahl, ${ }^{47}$ P. Stefko, ${ }^{48}$ O. Steinkamp, ${ }^{49,80}$ S. Stemmle, ${ }^{16}$ O. Stenyakin, ${ }^{43}$ H. Stevens, ${ }^{14}$ S. Stone, ${ }^{67}$ M. E. Stramaglia ${ }^{48}$ M. Straticiuc, ${ }^{36}$ D. Strekalina, ${ }^{80}$ S. Strokov, ${ }^{82}$ F. Suljik, ${ }^{62}$ J. Sun, ${ }^{26}$ L. Sun, ${ }^{72}$ Y. Sun, ${ }^{65}$ P. Svihra, ${ }^{61}$ P. N. Swallow, ${ }^{52}$ K. Swientek, ${ }^{34}$ A. Szabelski, ${ }^{35}$ T. Szumlak, ${ }^{34}$ M. Szymanski, ${ }^{47}$ S. Taneja, ${ }^{61}$ Z. Tang, ${ }^{3}$ T. Tekampe, ${ }^{14}$ F. Teubert, ${ }^{47}$ E. Thomas, ${ }^{47}$ K. A. Thomson, ${ }^{59}$ M. J. Tilley, ${ }^{60}$ V. Tisserand, ${ }^{9}$ S. T'Jampens, ${ }^{8}$ M. Tobin, ${ }^{6}$ S. Tolk, ${ }^{47}$ L. Tomassetti, ${ }^{20, f}$ D. Torres Machado, ${ }^{1}$ D. Y. Tou, ${ }^{12}$ M. Traill, ${ }^{58}$ M. T. Tran,${ }^{48}$ E. Trifonova, ${ }^{80}$ C. Trippl,${ }^{48}$ A. Tsaregorodtsev, ${ }^{10}$ G. Tuci, ${ }^{28, x}$ A. Tully, ${ }^{48}$ N. Tuning, ${ }^{31}$ A. Ukleja,${ }^{35}$ D. J. Unverzagt, ${ }^{16}$ A. Usachov, ${ }^{31}$ A. Ustyuzhanin,${ }^{41,81}$ U. Uwer, ${ }^{16}$ A. Vagner, ${ }^{82}$ V. Vagnoni, ${ }^{19}$ A. Valassi, ${ }^{47}$ G. Valenti ${ }^{19}$ N. Valls Canudas, ${ }^{44}$ M. van Beuzekom, ${ }^{31}$ H. Van Hecke, ${ }^{66}$ E. van Herwijnen,${ }^{80}$ C. B. Van Hulse, ${ }^{17}$ M. van Veghel,${ }^{77}$ R. Vazquez Gomez, ${ }^{45}$ P. Vazquez Regueiro, ${ }^{45}$ C. Vázquez Sierra, ${ }^{31}$ S. Vecchi, ${ }^{20}$ J. J. Velthuis, ${ }^{53}$ M. Veltri, ${ }^{21, z}$ A. Venkateswaran,${ }^{67}$ M. Veronesi, ${ }^{31}$ M. Vesterinen,${ }^{55}$ D. Vieira ${ }^{64}$ M. Vieites Diaz, ${ }^{48}$ H. Viemann, ${ }^{75}$ X. Vilasis-Cardona, ${ }^{44}$ E. Vilella Figueras, ${ }^{59}$ P. Vincent, ${ }^{12}$ G. Vitali, ${ }^{28}$ A. Vitkovskiy, ${ }^{31}$ A. Vollhardt, ${ }^{49}$ 
D. Vom Bruch, ${ }^{12}$ A. Vorobyev,${ }^{37}$ V. Vorobyev, ${ }^{42, e}$ N. Voropaev,${ }^{37}$ R. Waldi, ${ }^{75}$ J. Walsh,${ }^{28}$ C. Wang, ${ }^{16}$ J. Wang, ${ }^{3}$ J. Wang, ${ }^{72}$ J. Wang, ${ }^{4}$ J. Wang, ${ }^{6}$ M. Wang, ${ }^{3}$ R. Wang, ${ }^{53}$ Y. Wang, ${ }^{7}$ Z. Wang, ${ }^{49}$ D. R. Ward ${ }^{54}$ H. M. Wark, ${ }^{59}$ N. K. Watson, ${ }^{52}$ S. G. Weber, ${ }^{12}$ D. Websdale, ${ }^{60}$ C. Weisser, ${ }^{63}$ B. D. C. Westhenry, ${ }^{53}$ D. J. White, ${ }^{61}$ M. Whitehead, ${ }^{53}$ D. Wiedner, ${ }^{14}$ G. Wilkinson, ${ }^{62}$ M. Wilkinson, ${ }^{67}$ I. Williams, ${ }^{54}$ M. Williams, ${ }^{63,69}$ M. R. J. Williams, ${ }^{61}$ F. F. Wilson, ${ }^{56}$ M. Winn, ${ }^{11}$ W. Wislicki, ${ }^{35}$ M. Witek, ${ }^{33}$ L. Witola,${ }^{16}$ G. Wormser, ${ }^{11}$ S. A. Wotton, ${ }^{54}$ H. Wu, ${ }^{67}$ K. Wyllie,${ }^{47}$ Z. Xiang,,${ }^{5}$ D. Xiao, ${ }^{7}$ Y. Xie, ${ }^{7}$ H. Xing, ${ }^{71}$ A. Xu, ${ }^{4}$ J. Xu, ${ }^{5}$ L. Xu, ${ }^{3}$ M. Xu, ${ }^{7}$ Q. Xu, ${ }^{5}$ Z. Xu, ${ }^{5}$ Z. Xu, ${ }^{4}$ D. Yang, ${ }^{3}$ Y. Yang, ${ }^{5}$ Z. Yang, ${ }^{3}$ Z. Yang, ${ }^{65}$ Y. Yao, ${ }^{67}$ L. E. Yeomans, ${ }^{59}$ H. Yin, ${ }^{7}$ J. Yu, ${ }^{7}$ X. Yuan,${ }^{67}$ O. Yushchenko, ${ }^{43}$ K. A. Zarebski ${ }^{52}$ M. Zavertyaev ${ }^{15, w}$ M. Zdybal ${ }^{33}$ O. Zenaiev, ${ }^{47}$ M. Zeng, ${ }^{3}$ D. Zhang, ${ }^{7}$ L. Zhang, ${ }^{3}$ S. Zhang, ${ }^{4}$ Y. Zhang, ${ }^{47}$ Y. Zhang, ${ }^{62}$ A. Zhelezov, ${ }^{16}$ Y. Zheng, ${ }^{5}$ X. Zhou, ${ }^{5}$ Y. Zhou, ${ }^{5}$ X. Zhu, ${ }^{3}$ V. Zhukov, ${ }^{13,39}$ J. B. Zonneveld, ${ }^{57}$ S. Zucchelli, ${ }^{19, c}$ D. Zuliani, ${ }^{27}$ and G. Zunica ${ }^{61}$

\title{
(LHCb Collaboration)
}

\author{
${ }^{1}$ Centro Brasileiro de Pesquisas Físicas (CBPF), Rio de Janeiro, Brazil \\ ${ }^{2}$ Universidade Federal do Rio de Janeiro (UFRJ), Rio de Janeiro, Brazil \\ ${ }^{3}$ Center for High Energy Physics, Tsinghua University, Beijing, China \\ ${ }^{4}$ School of Physics State Key Laboratory of Nuclear Physics and Technology, Peking University, Beijing, China \\ ${ }^{5}$ University of Chinese Academy of Sciences, Beijing, China \\ ${ }^{6}$ Institute Of High Energy Physics (IHEP), Beijing, China \\ ${ }^{7}$ Institute of Particle Physics, Central China Normal University, Wuhan, Hubei, China \\ ${ }^{8}$ Univ. Grenoble Alpes, Univ. Savoie Mont Blanc, CNRS, IN2P3-LAPP, Annecy, France \\ ${ }^{9}$ Université Clermont Auvergne, CNRS/IN2P3, LPC, Clermont-Ferrand, France \\ ${ }^{10}$ Aix Marseille Univ, CNRS/IN2P3, CPPM, Marseille, France \\ ${ }^{11}$ Université Paris-Saclay, CNRS/IN2P3, IJCLab, Orsay, France \\ ${ }^{12}$ LPNHE, Sorbonne Université, Paris Diderot Sorbonne Paris Cité, CNRS/IN2P3, Paris, France \\ ${ }^{13}$ I. Physikalisches Institut, RWTH Aachen University, Aachen, Germany \\ ${ }^{14}$ Fakultät Physik, Technische Universität Dortmund, Dortmund, Germany \\ ${ }^{15}$ Max-Planck-Institut für Kernphysik (MPIK), Heidelberg, Germany \\ ${ }^{16}$ Physikalisches Institut, Ruprecht-Karls-Universität Heidelberg, Heidelberg, Germany \\ ${ }^{17}$ School of Physics, University College Dublin, Dublin, Ireland \\ ${ }^{18}$ INFN Sezione di Bari, Bari, Italy \\ ${ }^{19}$ INFN Sezione di Bologna, Bologna, Italy \\ ${ }^{20}$ INFN Sezione di Ferrara, Ferrara, Italy \\ ${ }^{21}$ INFN Sezione di Firenze, Firenze, Italy \\ ${ }^{22}$ INFN Laboratori Nazionali di Frascati, Frascati, Italy \\ ${ }^{23}$ INFN Sezione di Genova, Genova, Italy \\ ${ }^{24}$ INFN Sezione di Milano-Bicocca, Milano, Italy \\ ${ }^{25}$ INFN Sezione di Milano, Milano, Italy \\ ${ }^{26}$ INFN Sezione di Cagliari, Monserrato, Italy \\ ${ }^{27}$ Universita degli Studi di Padova, Universita e INFN, Padova, Padova, Italy \\ ${ }^{28}$ INFN Sezione di Pisa, Pisa, Italy \\ ${ }^{29}$ INFN Sezione di Roma Tor Vergata, Roma, Italy \\ ${ }^{30}$ INFN Sezione di Roma La Sapienza, Roma, Italy \\ ${ }^{31}$ Nikhef National Institute for Subatomic Physics, Amsterdam, Netherlands \\ ${ }^{32}$ Nikhef National Institute for Subatomic Physics and VU University Amsterdam, Amsterdam, Netherlands \\ ${ }^{33}$ Henryk Niewodniczanski Institute of Nuclear Physics Polish Academy of Sciences, Kraków, Poland \\ ${ }^{34}$ AGH-University of Science and Technology, Faculty of Physics and Applied Computer Science, Kraków, Poland \\ ${ }^{35}$ National Center for Nuclear Research (NCBJ), Warsaw, Poland \\ ${ }^{36}$ Horia Hulubei National Institute of Physics and Nuclear Engineering, Bucharest-Magurele, Romania \\ ${ }^{37}$ Petersburg Nuclear Physics Institute NRC Kurchatov Institute (PNPI NRC KI), Gatchina, Russia \\ ${ }^{38}$ Institute of Theoretical and Experimental Physics NRC Kurchatov Institute (ITEP NRC KI), Moscow, Russia \\ ${ }^{39}$ Institute of Nuclear Physics, Moscow State University (SINP MSU), Moscow, Russia \\ ${ }^{40}$ Institute for Nuclear Research of the Russian Academy of Sciences (INR RAS), Moscow, Russia \\ ${ }^{41}$ Yandex School of Data Analysis, Moscow, Russia \\ ${ }^{42}$ Budker Institute of Nuclear Physics (SB RAS), Novosibirsk, Russia \\ ${ }^{43}$ Institute for High Energy Physics NRC Kurchatov Institute (IHEP NRC KI), Protvino, Russia, Protvino, Russia \\ ${ }^{44}$ ICCUB, Universitat de Barcelona, Barcelona, Spain
}




\footnotetext{
${ }^{45}$ Instituto Galego de Física de Altas Enerxías (IGFAE), Universidade de Santiago de Compostela, Santiago de Compostela, Spain

${ }^{46}$ Instituto de Fisica Corpuscular, Centro Mixto Universidad de Valencia-CSIC, Valencia, Spain

${ }^{47}$ European Organization for Nuclear Research (CERN), Geneva, Switzerland

${ }^{48}$ Institute of Physics, Ecole Polytechnique Fédérale de Lausanne (EPFL), Lausanne, Switzerland

${ }^{49}$ Physik-Institut, Universität Zürich, Zürich, Switzerland

${ }^{50}$ NSC Kharkiv Institute of Physics and Technology (NSC KIPT), Kharkiv, Ukraine

${ }^{51}$ Institute for Nuclear Research of the National Academy of Sciences (KINR), Kyiv, Ukraine

${ }^{52}$ University of Birmingham, Birmingham, United Kingdom

${ }^{53}$ H.H. Wills Physics Laboratory, University of Bristol, Bristol, United Kingdom

${ }^{54}$ Cavendish Laboratory, University of Cambridge, Cambridge, United Kingdom

${ }^{55}$ Department of Physics, University of Warwick, Coventry, United Kingdom

${ }^{56}$ STFC Rutherford Appleton Laboratory, Didcot, United Kingdom

${ }^{57}$ School of Physics and Astronomy, University of Edinburgh, Edinburgh, United Kingdom

${ }^{58}$ School of Physics and Astronomy, University of Glasgow, Glasgow, United Kingdom

${ }^{59}$ Oliver Lodge Laboratory, University of Liverpool, Liverpool, United Kingdom

${ }^{60}$ Imperial College London, London, United Kingdom

${ }^{61}$ Department of Physics and Astronomy, University of Manchester, Manchester, United Kingdom

${ }^{62}$ Department of Physics, University of Oxford, Oxford, United Kingdom

${ }^{63}$ Massachusetts Institute of Technology, Cambridge, Massachusetts, USA

${ }^{64}$ University of Cincinnati, Cincinnati, Ohio, USA

${ }^{65}$ University of Maryland, College Park, Maryland, USA

${ }^{66}$ Los Alamos National Laboratory (LANL), Los Alamos, New Mexico, USA

${ }^{67}$ Syracuse University, Syracuse, New York, USA

${ }^{68}$ Laboratory of Mathematical and Subatomic Physics, Constantine, Algeria

[associated with Universidade Federal do Rio de Janeiro (UFRJ), Rio de Janeiro, Brazil]

${ }^{69}$ School of Physics and Astronomy, Monash University, Melbourne, Australia

(associated with Department of Physics, University of Warwick, Coventry, United Kingdom)

${ }^{70}$ Pontifícia Universidade Católica do Rio de Janeiro (PUC-Rio), Rio de Janeiro, Brazil

[associated with Universidade Federal do Rio de Janeiro (UFRJ), Rio de Janeiro, Brazil]

${ }^{71}$ Guangdong Provencial Key Laboratory of Nuclear Science, Institute of Quantum Matter, South China Normal University,

Guangzhou, China (associated with Center for High Energy Physics, Tsinghua University, Beijing, China)

${ }^{72}$ School of Physics and Technology, Wuhan University, Wuhan, China

(associated with Center for High Energy Physics, Tsinghua University, Beijing, China)

${ }^{73}$ Departamento de Fisica, Universidad Nacional de Colombia, Bogota, Colombia

(associated with LPNHE, Sorbonne Université, Paris Diderot Sorbonne Paris Cité, CNRS/IN2P3, Paris, France)

${ }^{74}$ Universität Bonn-Helmholtz-Institut für Strahlen und Kernphysik, Bonn, Germany

(associated with Physikalisches Institut, Ruprecht-Karls-Universität Heidelberg, Heidelberg, Germany)

${ }^{75}$ Institut für Physik, Universität Rostock, Rostock, Germany

(associated with Physikalisches Institut, Ruprecht-Karls-Universität Heidelberg, Heidelberg, Germany)

${ }^{76}$ INFN Sezione di Perugia, Perugia, Italy (associated with INFN Sezione di Ferrara, Ferrara, Italy)

${ }^{77}$ Van Swinderen Institute, University of Groningen, Groningen, Netherlands

(associated with Nikhef National Institute for Subatomic Physics, Amsterdam, Netherlands)

${ }^{78}$ Universiteit Maastricht, Maastricht, Netherlands

(associated with Nikhef National Institute for Subatomic Physics, Amsterdam, Netherlands)

${ }^{79}$ National Research Centre Kurchatov Institute, Moscow, Russia
}

[associated with Institute of Theoretical and Experimental Physics NRC Kurchatov Institute (ITEP NRC KI), Moscow, Russia]

${ }^{80}$ National University of Science and Technology "MISIS", Moscow, Russia

[associated with Institute of Theoretical and Experimental Physics NRC Kurchatov Institute (ITEP NRC KI), Moscow, Russia]

${ }^{81}$ National Research University Higher School of Economics, Moscow, Russia

(associated with Yandex School of Data Analysis, Moscow, Russia)

${ }^{82}$ National Research Tomsk Polytechnic University, Tomsk, Russia

[associated with Institute of Theoretical and Experimental Physics NRC Kurchatov Institute (ITEP NRC KI), Moscow, Russia]

${ }^{83}$ University of Michigan, Ann Arbor, Michigan, USA (associated with Syracuse University, Syracuse, New York, USA)

${ }^{a}$ Also at Laboratoire Leprince-Ringuet, Palaiseau, France.

${ }^{\mathrm{b}}$ Also at Università di Genova, Genova, Italy.

${ }^{\mathrm{c} A l s o}$ at Università di Bologna, Bologna, Italy.

${ }^{\mathrm{d}}$ Also at Università di Modena e Reggio Emilia, Modena, Italy.

${ }^{\mathrm{e}}$ Also at Novosibirsk State University, Novosibirsk, Russia.

${ }^{\mathrm{f}}$ Also at Università di Ferrara, Ferrara, Italy. 
${ }^{\mathrm{g}}$ Also at Università di Milano Bicocca, Milano, Italy.

${ }^{\mathrm{h}}$ Also at DS4DS, La Salle, Universitat Ramon Llull, Barcelona, Spain.

${ }^{\mathrm{i}}$ Also at Universidad Nacional Autonoma de Honduras, Tegucigalpa, Honduras.

${ }^{\mathrm{j}}$ Also at Università di Bari, Bari, Italy.

${ }^{\mathrm{k}}$ Also at Università di Cagliari, Cagliari, Italy.

${ }^{1}$ Also at INFN Sezione di Trieste, Trieste, Italy.

${ }^{\mathrm{m}}$ Also at Università degli Studi di Milano, Milano, Italy.

${ }^{\mathrm{n}}$ Also at Università di Roma Tor Vergata, Roma, Italy.

${ }^{\circ}$ Also at Universidade Federal do Triângulo Mineiro (UFTM), Uberaba-MG, Brazil.

${ }^{\mathrm{p}}$ Also at AGH-University of Science and Technology, Faculty of Computer Science, Electronics and Telecommunications, Kraków, Poland.

${ }^{\mathrm{q}}$ Also at Università di Siena, Siena, Italy.

${ }^{\mathrm{r}}$ Also at Università di Padova, Padova, Italy.

${ }^{\mathrm{s}}$ Also at Scuola Normale Superiore, Pisa, Italy.

${ }^{\mathrm{t}}$ Also at MSU-Iligan Institute of Technology (MSU-IIT), Iligan, Philippines.

${ }^{\mathrm{u}}$ Also at Università di Firenze, Firenze, Italy.

${ }^{v}$ Also at Hanoi University of Science, Hanoi, Vietnam.

${ }^{w}$ Also at P.N. Lebedev Physical Institute, Russian Academy of Science (LPI RAS), Moscow, Russia.

${ }^{\mathrm{x}}$ Also at Università di Pisa, Pisa, Italy.

${ }^{y}$ Also at Università della Basilicata, Potenza, Italy.

${ }^{\mathrm{z}}$ Also at Università di Urbino, Urbino, Italy. 